\title{
Immagini di Maria nella pittura e nei mosaici romani dalla crisi monotelita agli inizi della seconda iconoclastia (640-819)
}

\begin{abstract}
L'articolo analizza le immagini della Vergine, pittoriche e musive, presenti a Roma dalla metà del VII secolo ai primi decenni del IX. Il taglio prescelto cerca di usare in parallelo le diverse chiavi interpretative proposte dalla storiografia: l'analisi storica, le ricerche iconografico-iconologiche, le letture stilistico-formali. Nelle rappresentazioni di Maria si riflettono le dinamiche religiose dello sviluppo dottrinale e della pratica devozionale, legate prima alla eresia monotelita poi alla crisi iconoclasta; in seguito in esse convergono nuovi significati politici connessi alla nascente ideologia dello Stato della Chiesa. Lo sviluppo delle immagini mariane mostra nel corso dei quasi duecento anni esaminati il progressivo distacco dalla cultura bizantina ed il profilarsi di una nuova identità culturale.
\end{abstract}

Questo studio è dedicato alle raffigurazioni mariane realizzate a Roma tra il pontificato di Giovanni IV (640-642) e i primi anni di quello di Pasquale I (817-819).

Perché la scelta di questi limiti cronologici? Essi segnano due fasi di particolare significato nella evoluzione storica ed artistica romana. I due anni del dalmata Giovanni IV costituiscono nella prima metà del VII secolo l'avvio di un processo di distacco da Bisanzio: la politica religiosa imperiale a sostegno della eresia monotelita crea uno scontro con Roma ma negli anni di Giovanni IV e dei suoi successori la vita nella città è ancora profondamente legata all'oriente bizantino. Il pontificato del romano Pasquale I, iniziato nell'817 tre anni dopo la morte di Carlo Magno, coincide dal punto di vista politico con la fase matura dell'età carolingia. Il nuovo organismo dello Stato della Chiesa ha trovato una libera collaborazione con la dinastia franca e dal punto di vista artistico ha in parte sciolto i legami con Costantinopoli.

In che modo analizzeremo le immagini? Cercheremo di ricostruire i nessi tra iconografia, contesti politico-religiosi e soluzioni formali tentando di individuare alcune linee guida nell'evoluzione del fenomeno artistico a Roma nell'alto medioevo. 


\section{Maria orante nell'oratorio di San Venanzio in Laterano}

I mosaici che coprono la piccola calotta e l'arco absidale dell'oratorio di San Venanzio nel battistero lateranense (Fig.1) furono commissionati da Giovanni IV: il pontefice, ricordato nell'epigrafe che corre alla base del catino ${ }^{1}$, é rappresentato all'estrema destra del mosaico con il modello della chiesa tra le mani. Sappiamo che la cappella accolse le reliquie di dieci martiri dalmati, tra questi Venanzio, omonimo del padre di Giovanni, sottratte dal prete Martino a Salona alle scorrerie degli slavi e degli àvari. ${ }^{2}$ Sul lato opposto del mosaico, a sinistra, Teodoro chiude la decorazione: il papa, greco di Gerusalemme, nei suoi sette anni di regno (642-649) fece terminare i lavori.

Il mosaico absidale sviluppa in modo nuovo gli schemi teofanici consueti alla tradizione romana: duplica il registro compositivo e moltiplica il numero dei personaggi. Il centro visivo e concettuale è costituito dall'asse centrale CristoMaria: in alto il busto del Redentore in mezzo a nubi infuocate benedice alla greca tra due angeli acclamanti, nel piano inferiore Maria in posa di orante, i principi degli apostoli, i due Giovanni (titolari delle cappelle omonime nel battistero), i martiri della Dalmazia ed i due papi committenti. ${ }^{3}$ Nell'imposta dell'arco absidale prosegue la teoria dei martiri in due pannelli di quattro figure ciascuno.

Dedichiamoci in via preliminare a comprendere il carattere compositivo nel suo insieme, osserviamo in secondo luogo la figura di Maria, ed infine proviamo a leggere le soluzioni formali.

Lo schema generale si pone come una sintesi di elementi romani e di prestiti esterni. La successione tradizionale romana dei martiri titolari e dei papi committenti si "contamina" nella raffigurazione dell'Eterno con i precedenti di soggetti apocalittici e si arricchisce nella duplicazione del registro e nella assialità Vergine-Redentore con i modelli dell'Ascensione provenienti dall'oriente siro-pa-

1. MARTYRIBUS XPI DNI PIA VOTA JOHANNE/ REDDIDIT ANTISTES SANCTIFICANTE DO/ AC SACRI FONTIS SIMILI FULGENTE METALLO/ PROVIDUS INSTANTER HOC COPULAVIT OPUS/ QUO QUISQUIS GRADIENS ET XPM PRONUS ADORANS EFFUSASQUE PRECES MITTAT AD AETHRA SUAS. Sull'iscrizione si veda De Rossi 1872, pagine non numerate. Sull'oratorio si veda: Armellini 1942, I, 136; II,1306; Pelliccioni 1973.

2. Johannes, natione Dalmata, ex patre Venantio scolastico [...] temporibus suis misit per omnem Dalmatiam seu Histriam multas pecunias per sactissimum et fidelissimum Martinum abbatem propter redemptionem captivorum qui depraedati erant a gentibus. Eodem tempore feci ecclesiam beatis martyribus Venantio, Anastasio, Mauro, et aliorum multorum martyrum quorum reliquias de Dalmatias et Histrias adduci praeceperat, et recondit eas in ecclesia suprascripta [...], L. P., I, LXXIIII, 124. Sulla missione di Martino si veda anche Monumenta Spectantia XIV, III, 1894, 28-29.

3. Sullo schema teofanico di tradizione romana definitosi nel V secolo si veda Matthiae 1967, 131-133, 138-139. 


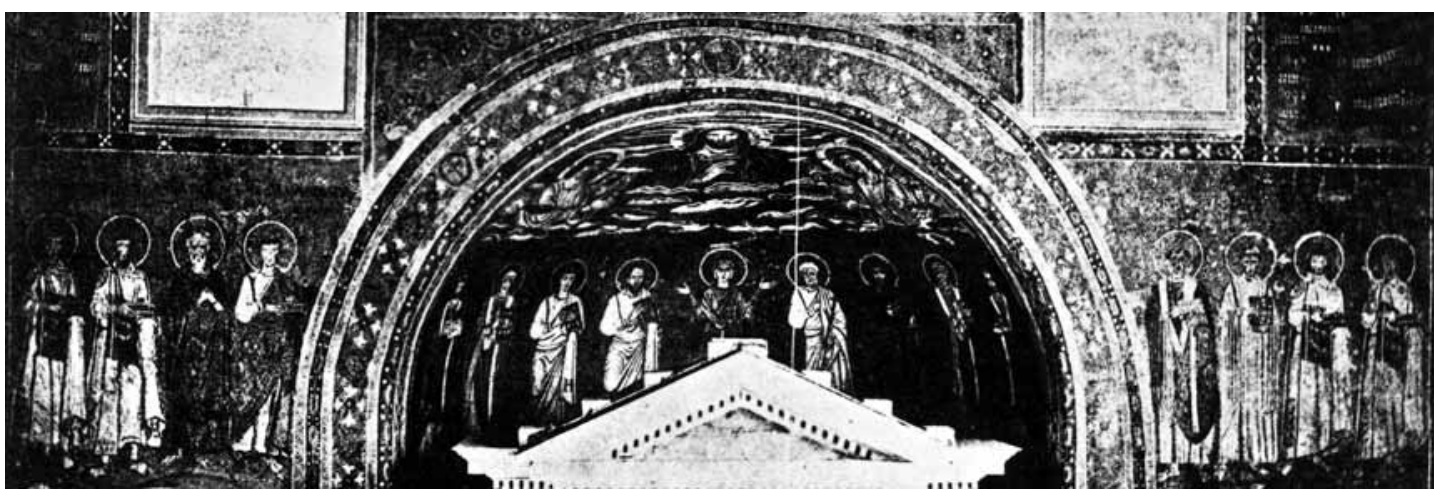

Fig. 1. Mosaico dell'abside, battistero di San Giovanni in Laterano, cappella di San Venanzio (Foto ICCD, serie F, neg. 73268)

lestinese e copto. ${ }^{4}$ Anche la continuazione semantica della decorazione absidale nell'arco -la teoria dei martiri prosegue nei due pannelli di imposta- costituisce un elemento estraneo agli usi romani, frequente invece nelle visioni teofaniche dei centri copti ${ }^{5}$.

Soffermiamoci su Maria (Fig. 2) che ripropone nella figura frontale con le braccia aperte a compasso l'antico gesto di preghiera dei primi cristiani: essa è colei che rivolge la domanda di salvezza. ${ }^{6}$ Abbiamo visto la sua collocazione nel contesto dell'Ascensione in ambito siro-palestinese e copto dove viene talvolta sostituita dalle immagini della Vergine Madre di Dio in trono. ${ }^{7}$ Nella forma isolata trovava a Costantinopoli il suo "tipo aulico" nella immagine detta Episkepsis nel perduto santuario delle Blachernae: l'icona della Vergine in figura

4. Si vedano per gli antecedenti apocalittici romani: Matthiae 1967, I, 60; Andaloro 1987, 232. Per i modelli copti si rimanda ai lavori pionieristici dell'Istituto Archeologico francese del Cairo: Clédat 1904, 517; Clédat 1904-1906, 75 con fig. 148; al fondamentale Grabar 1946, 192 ed alle ultime sintesi storiografiche: Velmans et al.1999, 55-62; Iacobini 2000, 43-65. Per i modelli siro palestinesi si rinvia a Grabar 1958, 26-27; tvv. XVII, XIX, XXI; Lazarev 1967, 42. Sul rapporto tra San Venanzio e gli affreschi copti si veda anche Matthiae 1967, 192. Per la lettura iconografica specifica di San Venanzio si rinvia soprattutto a: Grabar 1946, 58; Grabar 1968, 132.
5. Grabar 1946, 116. La parete presbiteriale sud di S. Maria Antiqua presenta nelle figure dei pontefici Martino I (649-653) e Giovanni VII (702-705) una anomala estensione della perduta decorazione absidale dell'età di Giovanni VII: Nordhagen 1968, 94.

6. Cecchelli individua Maria orante con il Bambino in esempi cimiteriali di IV secolo: Cecchelli 1946, 133-135 con figure dell'A.

7. Sulle origini dell'iconografia dell'Ascensione nell'ambiente siro-palestinese e sul successivo passaggio in Egitto si vedano: Grabar 1946, 177-180; 209-211; 233-237; Lazarev 1967, 89, 102 n. 50. Sui precoci esempi della Cappadocia si veda Thérel 1984, 42 con bibliografia. 
di orante aveva sul petto un clipeo con il Bambino. Un velo che la occultava si apriva e si chiudeva miracolosamente ${ }^{8}$.

Ripensiamo al momento storico in cui è nato il mosaico. Nel 638, quindi circa due anni prima dell'apertura del cantiere lateranense, l'imperatore Eraclio, in accordo con Sergio patriarca di Costantinopoli, fa affiggere nella capitale, nel nartece di S. Sofia, l'Ekthesis. L' antica disputa sul dogma della Incarnazione riemerge: la natura umana e divina di Cristo è negata dalla dottrina monoenergetica e divampa l'incendio della crisi monotelita. Se papa Onorio (625-638) aveva dato la sua approvazione, il successore Severino (638-640) si rifiuta di sottoscrivere l'Ekthesis e Giovanni IV condanna l'eresia monotelita nel sinodo del 641. Anche Teodoro tentò tra il 645 ed il 646 di ristabilire l'ortodossia9 .

Riflettiamo sul possibile il rapporto tra il mosaico di San Venanzio e le vicende politiche e teologiche. Al Redentore nei cieli dell'Empireo, Dio sub specie aeternitatis, fa riscontro Dio sub specie universi rappresentato dai testimoni terreni della Incarnazione: Maria in gesto di intercessione, gli apostoli ed i martiri ${ }^{10}$.

Soffermiamoci adesso sullo stile. Gli studi hanno sottolineato di volta in volta le componenti romane ${ }^{11}$, il rapporto assai stretto con le esperienze bizantine contemporanee dei mosaici di San Demetrio a Salonicco ${ }^{12}$ ed una parziale derivazione dagli affreschi frammentari del VI secolo nell'anfiteatro di Salona ${ }^{13}$.

Il complesso musivo risulta un episodio chiave della cultura artistica romana che ci appare come un fiume nel quale si vengono a mescolare acque di provenienza diversa. L'isolamento paratattico delle figure allineate sul fondo d'oro parla, come è stato notato, il nuovo linguaggio bizantino delle icone ${ }^{14}$; gli angeli e il Cristo benedicente sono romani nella accentuazione dello squadro volumetrico, le nove figure del registro inferiore della calotta alternano brevi echi di suggestioni plastiche a soluzioni decise verso l'astrazione che raggiunge la sua pienezza negli otto martiri dell'arco absidale.

8. Sulla Madonna col clipeo o Blachernitissa "tipo aulico della Maria orans" e sulle sue varianti, sulla sua ipotetica cronologia preiconoclasta: Cecchelli 1946, 214-220 con disegni dell'A.; Thérel 1984, 43; si veda anche Bisogni 2000, 166-167. Sulle connessioni con l'iconografia della Hagiosoritissa, sui legami concettuali tra Annunciazione, Incarnazione, Intercessione si veda Andaloro 1970, 118-119; 139.

9. Sulla crisi monotelita e sui riflessi a Roma nella prima metà del VII secolo si vedano: Grumel 1928; Grumel 1929; Grumel 1930; Bognetti 1955; Bertolini 1958, 733-739; Ostrogorsky 1968, 95-108; Conte 1971, 271-281; von Schönborn 1975, Zocca 1992; Burgarella 2002, in particolare 969-971 con bibliografia. Sul sinodo del 641: Mansi 1764, 607; sul sinodo degli anni 645-646: Mansi 1764, 610.

10. Grabar 1946, 58; Grabar 1968, 132; Themelly
1999, 319-328

11. Ricordiamo soprattutto: Matthiae 1967, I, 195; Nordhagen 1965, 155: lo studioso considera l'oratorio lateranense risultato di un cantiere locale per l'uso di sole tessere vitree.

12. Lazarev 1967, 98 n. 17; Oakeshott 1967, 117; Bovini 1971, 153-154; Andaloro 1987, 247; Andaloro 1992, 602; Kitzinger 1989, 118-121; Kitzinger 1992, 118-121.

13. Dyggve 1951, 84-87, fig. IV, 46, 47; Bovini 1971, 153-154. Sul rapporto con gli affreschi si veda Themelly 1999, 327.

14. Gli studi di Kitzinger chiariscono la funzione fondante ma non univoca svolta dal culto delle icone nella conquista del linguaggio astratto del VII secolo: Kitzinger 1989, soprattutto 118-119; Kitzinger 1992, in particolare 149-150; Andaloro 1987, 247; Andaloro 1992, 602. 


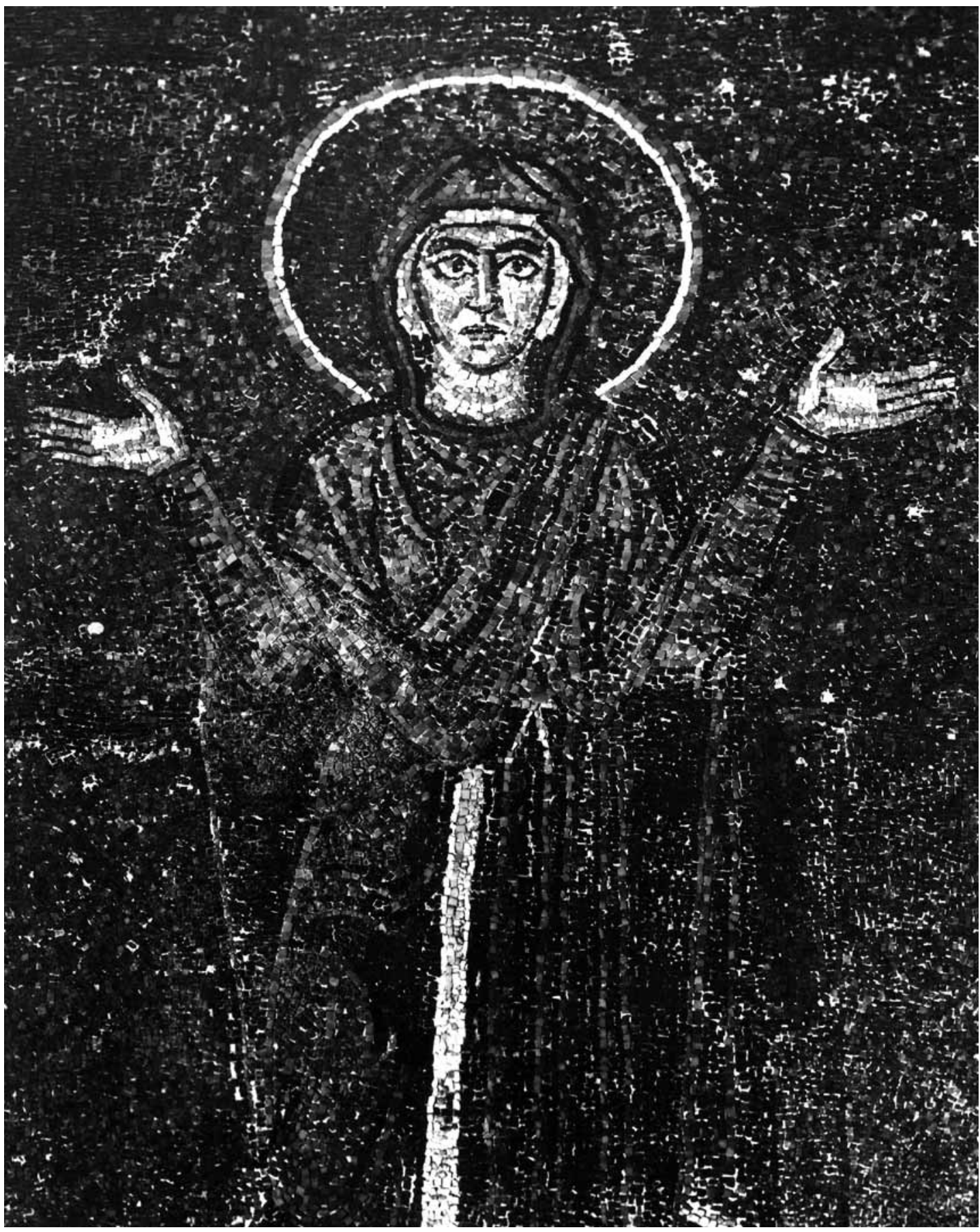

FIG. 2. Maria orante, battistero di San Giovanni in Laterano, cappella di San Venanzio (Foto ICCD, serie E, neg. 2293) 
Maria orante è un chiaro segno della compresenza di culture diverse. Rivela nella iconografia dal deciso apporto teologico il prestito dall'oriente bizantino e mostra nella risoluzione netta in favore di un nuovo linguaggio astratto la parentela stretta, ma non la coincidenza, con i coevi pannelli musivi in San Demetrio a Salonicco. Consideriamola in rapporto alla figura del Santo titolare realizzato nella chiesa greca nel pilastro ovest del bema ${ }^{15}$ : le due figure ci appaiono vicine e nello stesso diverse. Nello scarto bidimensionale dell'avambraccio in atteggiamento di preghiera "circola lo stesso sangue", ma nei due volti ci sembra di riconoscere "due fratelli in cui si mescolano razze diverse". San Demetrio ha il corpo astratto e medioevale ma nella faccia fiorisce di vita tridimensionale: le tessere di pietra, piccole ed eterogenee piegano il mosaico, secondo l'uso antico ancora vivo nel mondo greco, alle leggi del naturalismo. La figura di Maria è invece del tutto medioevale: le tessere solo vitree, affermatesi nella pratica romana a partire dal V secolo, omogenee nelle dimensioni e nel taglio rettangolare, uniformi nell'assorbimento della luce, rispondono nella distribuzione bidimensionale del colore alle nuove leggi dell'astrazione.

Solo la testa di Mauro e quella di Gaiano, nel pannello a destra dell'arco absidale, mostrano nelle tessere sottili ed irregolari che imitano faticosamente la tecnica bizantina e nel disporsi graduale del colore, una eco lontana dell'uso pittorico del mosaico ${ }^{16}$.

Appunti su alcune immagini della Vergine in S. Maria Antiqua: la difesa dell'ortodossia nei lasciti della devozione individuale

È impossibile occuparsi delle immagini di Maria nell'alto Medioevo romano senza soffermarsi, seppur brevemente e con cautela, su S. Maria Antiqua. Essa conservava l'imago antiqua ${ }^{17}$ ed un numero elevato di immagini di Maria.

La chiesa scoperta nei primi anni del '700 ma scavata solo nei primi del '900, continua tutt'oggi ad essere oggetto di riflessioni e di interrogativi ${ }^{18}$ : riemersa

15. Velmans et al. 1999, 19-20, figg. 7, 11. Sono fondamentali per la lettura formale: Lazarev 1967, 73-74; e per la nuova funzione dell'immagine: Grabar 1946, 119; Kitzinger 1989, 118-119; Kitzinger 1992, 156-172.

16. Dobbiamo a Nordhagen ed ai suoi studi dell'ultimo trentennio il nuovo indirizzo metodologico volto ad indagare le componenti formali associate alla lettura tecnica del mosaico romano di età pre-carolingia. Per l'ampia bibliografia dell'A. si rinvia a Nordhagen 1997, 574. Per le immagini delle teste di Mauro e di Gaiano si veda Matthiae 1967, II, figg. 117, 120. Per il riconoscimento di queste come parte originali del mosaico di VII secolo si rinvia al grafico dei restauri in Themelly 1999, fig. 15. Sulle vicende storiche dei restauri si veda: Curzi 1998.

17. Sulla icona ora nella Basilica di S. Maria Nova al Foro Romano e sulla bibliografia si veda Andaloro 2002, 723 n. 14, 745.

18. Impossibile in questa sede ricordare gli studi che le sono stati dedicati. Citiamo solo quelli che ci sembra abbiano determinato dei momenti essenziali di snodo critico: la lettura epigrafica di Rushfort 1902, le monografie di Grüneisen 1911 e di Tea 1937; gli studi di Kitzinger 1934; 1989, 127-137; le analisi a tappeto di Nordhagen, 1962a, 1968, 1978, lo studio di Osborne sull'atrio del 1987. Si vedano inoltre Brenk 2003, che attribuisce un nuovo ruolo a papa Teodoro, in particolare 1007, 1051-1053; e i saggi in S. Maria Antiqua al Foro Romano 2004. 
all'interno di un edificio pubblico romano ai piedi del Palatino ${ }^{19}$ ci ha consegnato una decorazione di qualità incredibilmente elevata, risolta in gran parte nella koiné del naturalismo antico.

Per diverse ragioni la chiesa riveste un ruolo eccezionale: getta luce sulle sopravvivenze dell'arte ellenistica a Roma. ${ }^{20}$ Esprime in modo esemplare la connessione profonda tra le immagini e le politiche religiose ufficiali: le sue immagini si legano infatti al concilio antimonotelita guidato da Martino I nel 649 in Laterano ed al concilio Trullano o Quinisesto tenutosi a Costantinopoli nel 692. ${ }^{21}$ Documenta inoltre, nella decorazione a pannelli iconici diffusi quasi a sistema, il manifestarsi, inedito per l'occidente, di nuove forme di devozione individuale. ${ }^{22}$

Ragioniamo sulle immagini di Maria. Limiteremo l'attenzione ai pannelli isolati con l'immagine della Vergine realizzati negli anni del pontificato dell'umbro Martino I (649-653) ${ }^{23}$ e in quelli del greco Giovanni VII (705-707).

Per il primo gruppo abbiamo otto raffigurazioni di Maria: tra le immagini predominano quelle legate al dogma dell'Incarnazione. Nell'area del santuario la ritroviamo nella parete presbiteriale destra, nel pannello con S. Anna ${ }^{24}$, e ai lati dell'abside: a destra nell'Annunciazione della parete palinsesto e a sinistra in trono con una figura di Santo ${ }^{25}$. I due primi pilastri meridionali accolgono una concentrazione di immagini. Abbiamo nel pilastro sud-est tre rappresentazioni: la Vergine con il Bambino con le mani incrociate, la cosiddetta Annunciazione di Martino I e la figurazione di Deesis con donatore ${ }^{26}$, e nel pilastro sud-ovest Maria tra angeli. ${ }^{27}$ La colonna nord-ovest accoglie infine la figurazione di Maria Eleousa. $^{28}$

Passiamo alla decorazione di Giovanni VII. La ricostruzione proposta da Grüneisen nel 1911 (Fig. 3) ha ipotizzato per l'abside Maria Regina tra due angeli e gli apostoli Pietro e Paolo. La Vergine con gli attributi regali avrebbe costituito una rielaborazione del tema originario decurtato dall'apertura della nicchia absidale. La diversa datazione al VII secolo proposta da Kitzinger nel 1934 in base all'analisi delle figure laterali superstiti ci lascia comunque nell'ambito delle

19. Sulla trasformazione in chiesa negli anni di Giustino II (565-578) dell'edificio già cristianizzato dalla metà del VI secolo e sulla originaria funzione di corpo di guardia: Krautheimer 1981, 93, 418.

20. Il recentissimo intervento di Brubaker si discosta dal parere comune degli studiosi e considera il naturalismo cosiddetto ellenistico di S. Maria Antiqua un prodotto romano e non una "derivazione bizantina”, Brubaker 2004, in particolare 44.

21. Si vedano soprattutto Rushfort 1902, 72; Nordhagen 1962a, 58-59; Nordhagen 2002; Lindsday Opie 2002.

22. Si vedano essenzialmente: Grabar 1946, 102;
Nordhagen 1978, 136-138; Kitzinger 1992, 190 191; Sansterre 1997, 112; Bauer 1999, 410-411.

23. Per l'individuazione sulla base dell'analisi degli intonaci della campagna decorativa di Martino I si rimanda a Nordhagen 1962a, 58-61.

24. Nordhagen 1978, 100-101, fig. I, e.

25. Nordhagen 1978, 93-97, fig. I, a, b.

26. Nordhagen 1978, 103-105, 107-111, fig. I, f, h, i. Si rimanda per la Vergine con le mani incrociate a Nordhagen 1963; per la Deesis a Nordhagen 2000.

27. Nordhagen 1978, 122, fig. I, l.

28. Nordhagen 1978, 130-132, fig. I, t. Sull'iconografia dell'Eleousa: Nordhagen 1962b. 


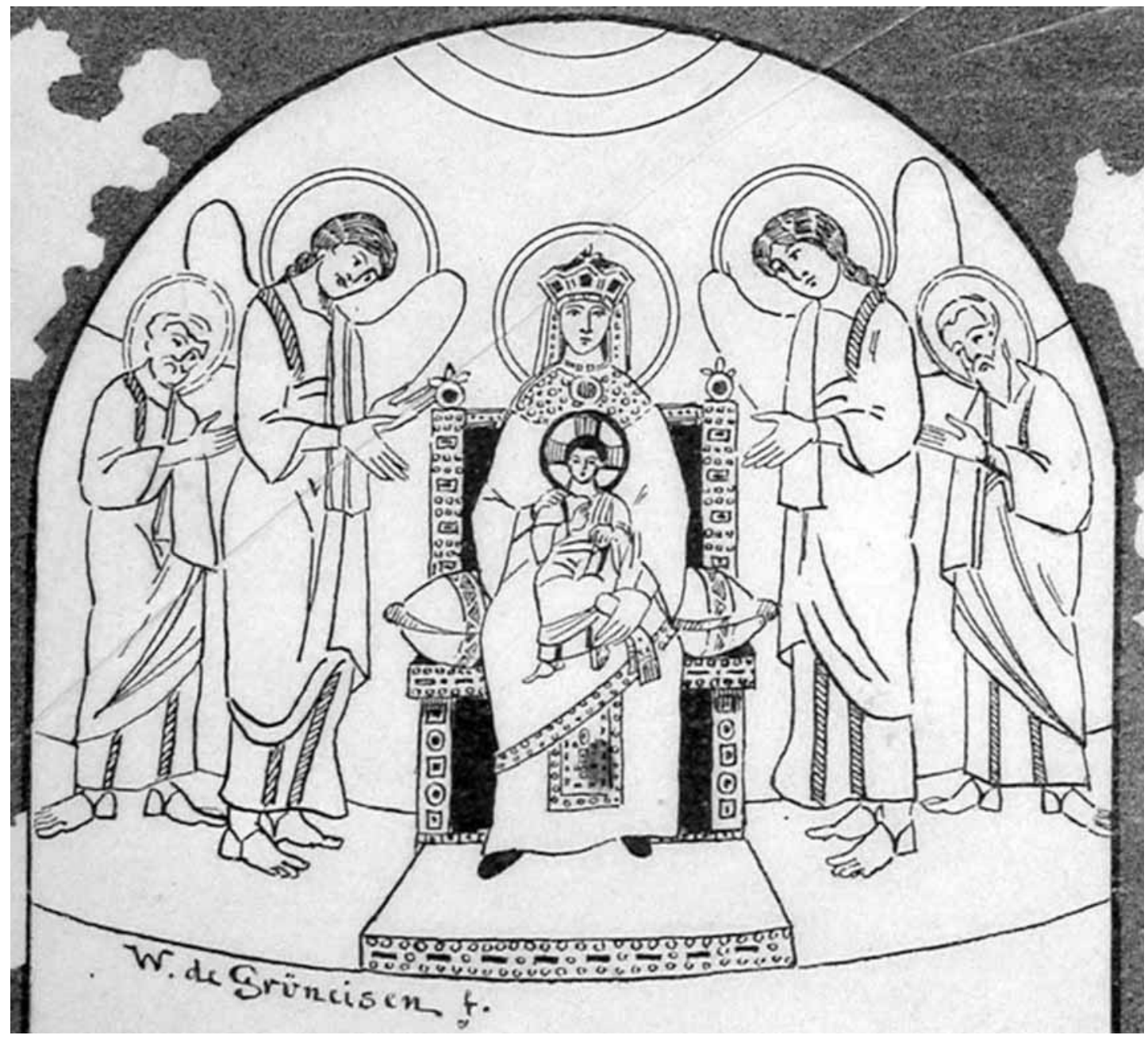

FIG. 3. Abside di Giovanni VII nella ricostruzione di Grüneisen, 1911 (Foto BIASA)

ipotesi per quanto riguarda la presenza o meno al centro dell'abside di Maria Regina. $^{29}$

Il ciclo presbiteriale della vita di Cristo, che non prendiamo in considerazione, si sofferma ripetutamente sui temi dell'Infanzia. ${ }^{30}$ Le immagini isolate di Maria sono sei e si estendono fino nell'atrio. Nella parete orientale del presbiterio un

"Le titre de la basilique rend très probable que cette place etait réservée à Marie-Reine”: Grüneisen 1911, 142, 306-307; sulla presenza ipotetica di Maria Regina si vedano anche: Tea 1937, 305; Meer 1938, 324; Ihm 1960, 58 fig. 11, nr. XI, 145-146; Wisskirchen 1995-1997, 385, 391 n. 77. Per l'attribuzione dei soli frammenti superstiti al VII secolo: Kitzinger 1934, 42; Nordhagen 1978, 92-93 fig. XIV a,b; sull'abside di Giovanni VII "priva di tracce figurative significanti”: Nordhagen 1968, 54.

30. Grabar 1946, 129 e sgg.; Nordhagen 1968, 90-91. 
incavo incorniciava una immagine di Maria. ${ }^{31}$ La seconda Annunciazione venne a coprire nel pilastro sud-est quella di VII secolo. ${ }^{32}$ Il pilastro nord-ovest accoglie una nicchia con l'immagine di Maria con il Bambino ${ }^{33}$ (Fig. 4). A fianco della porta che conduce alla rampa che sale al Palatino troviamo Maria con il Bambino, i Santi Pietro e Paolo e un donatore. ${ }^{34}$ La seconda nicchia della parete sinistra dell'atrio presenta Maria tra due donatori. ${ }^{35}$ Sulla facciata dell'oratorio dei Quaranta martiri infine c'è l'immagine di Maria in trono tra gli angeli ed il donatore (Giovanni VII?). ${ }^{36}$ Sotto Giovanni VII registriamo un'attenzione rivolta alla rappresentazione di Maria Madre di Dio coniugata ad una crescita delle immagini devozionali.

Le pagine scritte da Nordhagen illuminano sull'analisi della decorazione pittorica: lo studioso individua nell'uniformità degli intonaci l'appartenenza degli affreschi ad una unitaria campagna della metà del VII secolo: all'interno del comune denominatore "ellenistico", esponenti di differenti stili lavorano fianco a fianco esprimendo diversi gradi di naturalismo: le forme più pure si costruiscono con le sole variazioni del colore senza l'uso della linea. ${ }^{37}$

È con il cantiere di Giovanni VII che assistiamo ad una evoluzione: la consistente introduzione degli elementi lineari, l'accentuazione espressiva dei gesti e delle fisionomie, la riduzione della illusione della profondità spaziale alterano ma non negano la costruzione classica della forma. ${ }^{38}$

Le immagini mariane che abbiamo considerato sottolineano costantemente il credo duofisita ${ }^{39}$. Riflettiamo su questi dati. Il VII secolo assiste ad una esplosione delle eresie che negano la dottrina della Incarnazione. Il concilio lateranense del 649 ed il concilio di Toledo del 693 segnano due tappe importanti nella difesa della ortodossia: il dogma viene ribadito anche mediante l'affermazione della verginità-maternità di Maria. La condanna del monofisismo viene proclamata a Bisanzio nel VI concilio ecumenico del 680 e nel concilio Quinisesto o Trullano degli anni 691-692. Malgrado ciò l'eresia monotelita nel primo decennio dell'VIII secolo era ancora attiva ${ }^{40}$.

31. Nordhagen 1968, 17; Bauer 1999, 410.

32. Nordhagen 1968, 78-79, fig. CXXXII, a.

33. Nordhagen 1968; 75-76, fig. CXXXII, 6; Bauer 1999, 411.

34. Nordhagen 1968, 80-81, CXXXII, e.

35. Nordhagen 1968, 83, CXXXII, 8. Per una datazione più tarda si veda Osborne 1987, 197-198.

36. Nordhagen 1968, 84-85, CXXXII, 9.

37. Nordhagen 1978, in particolare 138-140; si vedano anche le magistrali letture di Kitzinger 1989, 127-135, che riconduce le variazioni stilistiche a differenti cronologie. Per le possibili presenze di artisti romani a contatto con gli artisti costantinopolitani si veda Lazarev 1967,
70; Krautheimer 1981, 135.

38. Nordhagen 1978: 138-140; Nordhagen 1968: 101-114. Per una sintesi: Nordhagen 1983.

39. Sul programma mariano: Nordhagen 1968, in particolare 89-93; Brenk 2004, in particolare 77.

40. Sul concilio del 649: Mansi 1764, 863-1170, in particolare 965-966; 991-994. Sul concilio di Toledo del 693: Mansi 1766: 59-87, in particolare: 63-68. Per i concili costantinopolitani e per le persistenze dell'eresia monotelita nell'età di Giovanni VII si rinvia a Ostrogorsky 1968, 111, 119,141 . Si veda per un sintetico ma efficace quadro complessivo della mariologia nell'alto medioevo latino: D’Onofrio 2000, 56-63. 


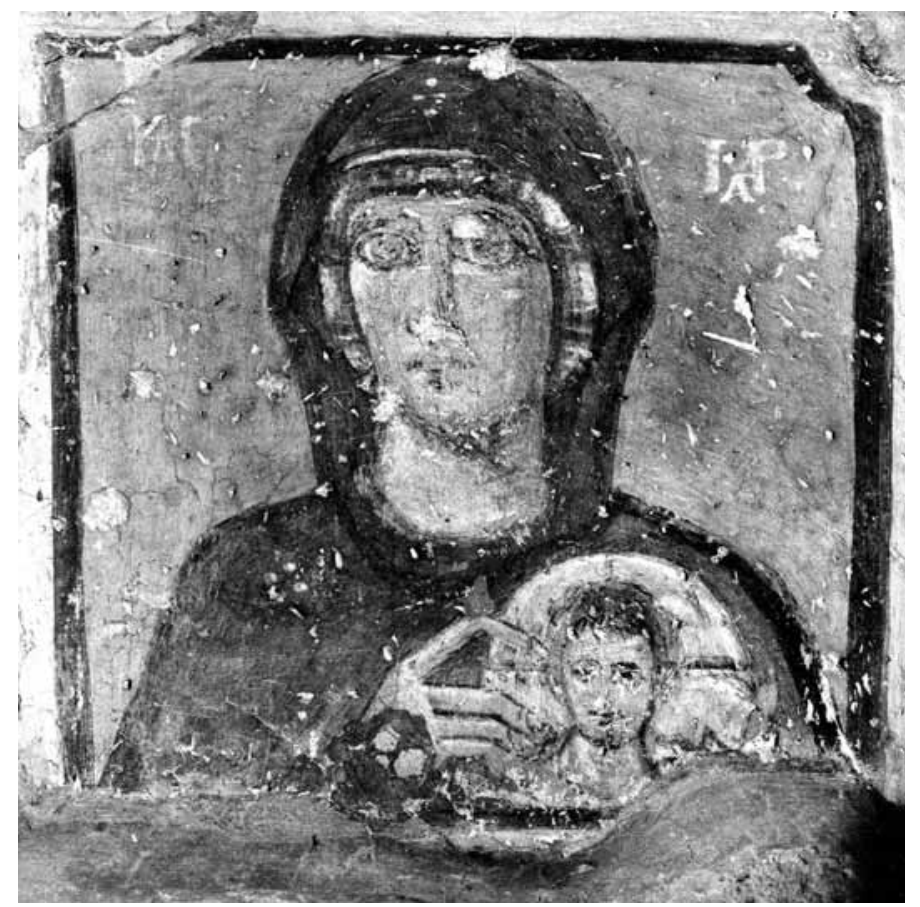

FIg. 4. Maria con il Bambino, Roma, Santa Maria Antiqua, pilastro nord-ovest (Foto Soprintendenza Speciale per il Polo Museale, neg. 197502)

L'intensificarsi a Roma della devozione mariana a partire dal VII secolo è un altro aspetto della stessa tendenza: Sergio I (687-701) introduce le festività della Dormizione, dell'Annunciazione e della Natività. ${ }^{41}$ Con Giovanni VII assistiamo ad un ulteriore intensificarsi del culto: il papa sposta la residenza papale sul Palatino, nell'area della Domus Tiberiana, o nell'Atrium Vestae, nei pressi di S. Maria Antiqua; realizza per la Vergine l'oratorio mariano in San Pietro in Vaticano (Fig. 5) e la Icona della Clemenza in Santa Maria in Trastevere. ${ }^{42}$

Sansterre e Burgarella hanno esemplarmente chiarito il quadro storico-culturale della città di Roma nel VII e nell'VIII secolo aperta alle presenze "greche". Secondo Burgarella i nuclei di ellenofoni, provenienti dall'oriente palestinese, siriaco e greco, dalle province imperiali dell'Italia meridionale ed insulare, avversi alla politica religiosa imperiale e sostenitori intransigenti dell'ortodossia, erano favoriti dai meccanismi del reclutamento ecclesiastico: la loro conoscenza della politica, degli affari diplomatici, e della lingua li rendeva forte-

41. Bertolini 1941, p. 407; Sensi 2000, 98-99.

42. Su Giovanni VII si veda per l'operato politico ed il rimando alle fonti: Bertolini 1941, 410-412; per una analisi del suo mecenatismo: Krautheimer 1981, 132; sullo spostamento dell'episcopio e sulla sua portata politica: Augenti 1996, 56, 60. Sulla Icona della Clemenza: Bertelli 1964; Andaloro 1972-1973 con una datazione al VI secolo; Andaloro 2002, 725-731. Sui mosaici frammentari dell'oratorio: Nordhagen 1965; Andaloro 1989. 

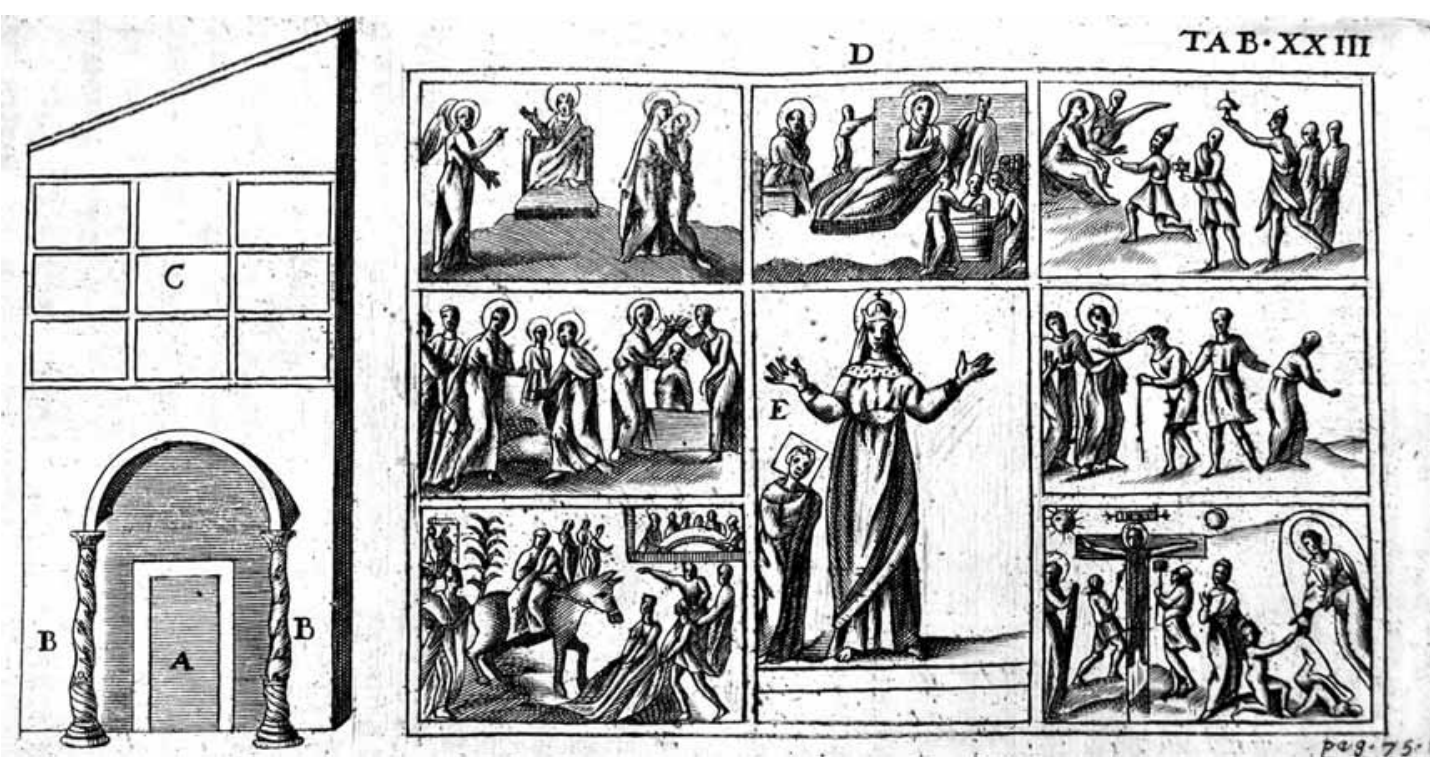

FIG.5. L'oratorio vaticano di Giovanni VII, Ciampini, De sacris aedificiis, Roma 1699, tv. XXIII (Foto BIASA)

mente competitivi tanto da far loro raggiungere un ruolo egemone nell'anno del concilio antimonotelita ${ }^{43}$.

Sansterre accogliendo la posizione di una parte della storiografia ha ritenuto che la chiesa ai tempi di Giovanni VII e con probabilità sin dal VII secolo fosse già il santuario di un istituto diaconale: contigua al quartiere più ellenizzato della città, frequentata dai funzionari imperiali che risiedevano sul Palatino secondo lo studioso aveva probabilmente il servizio liturgico ed assistenziale assicurato da monaci greci. Negli anni della crisi monotelita S. Maria Antiqua fu il teatro dello scontro tra Roma e Bisanzio e nello stesso tempo il luogo del clima di collaborazione che si venne a creare tra l'amministrazione bizantina locale - l'esarca ribelle Olimpio - la chiesa ed i gruppi monastici. Essi contribuirono largamente alla direzione teologica ad alla redazione degli atti del concilio, che fu in greco, e secondo lo studioso non furono estranei al programma decorativo del muro absidale della chiesa che presenta, seguendo l'identificazione proposta da Rushfort nel 1902, quattro Padri che sorreggono rotuli contenenti loro passi emblematici relativi al credo duofisita letti al concilio ${ }^{44}$. Brenk di recente ha sviluppato in modo suggestivo questa posizione suggerendo di individuare in S.Maria Antiqua il luogo di incontro ufficiale tra l'amministrazione bizantina ed i monaci greci in arrivo, ed in questi ultimi i possibili committenti degli ex voto ${ }^{45}$.

43. Burgarella 2002, in particolare 965-969.

44. Sansterre 1983 I, 9, 62-63, 105-106, 109, 117119, 126; II, 145-148, 161-162.

45. Brenk 2004, in particolare 79. Sull'identifica- zione degli ex voto in base alla presenza del donatore o delle iscrizioni di carattere votivo si veda Nordhagen 1978, 141-142. 
Sottolineiamo che se la decorazione di committenza papale esprime in messaggi ufficiali l'impegno dottrinale di quegli anni, anche la devozione individuale, sia essa di monaci o di laici, formula, anche se in modo meno organico, una risposta alle tensioni religiose del momento. Se i Padri della Chiesa della parete palinsesto, oggi parte frammentaria di un articolato piano decorativo dell'area presbiteriale ed absidale dell'età di Martino I, citano nei rotuli le sottili esposizioni dottrinali sulle due nature congiunte in Cristo le "icone" di Maria ribadiscono lo stesso credo.

Consideriamo infine che l'opposizione a Bisanzio si esprime in una lingua "greca", la separazione è religiosa, non ancora né politica né culturale.

\section{Maria Regina a Roma nell'VIII secolo e l'eclissi di Bisanzio}

L'arte a Roma nell'VIII secolo accorda una preferenza particolare al soggetto di Maria Regina. Comparso come un astro nel VI secolo in S. Maria Antiqua nella celebre parete palinsesto rimane esempio unico a Roma fino all'età di Giovanni VII. Abbiamo visto come le ripetute immagini mariane del VII secolo nella chiesa del Foro prediligano la rappresentazione della Vergine, Madre di Dio, con una presenza saltuaria e parziale degli attributi celesti degli angeli e regali del trono gemmato. È l'età di Giovanni VII che segna il successo di questa iconografia. Le immagini imponenti fatte realizzare dal pontefice esaltano la regalità della Vergine sottolineandone la natura di Madre di Dio. L'iscrizione dell'icona di S. Maria in Trastevere allude alla nascita divina dal ventre di Maria ${ }^{46}$ il frammento musivo dell'oratorio vaticano ora a Firenze ripropone Maria Regina testimone dell'Incarnazione in posa di orante. ${ }^{47}$

Nel corso del secolo il soggetto si diffonde: compare in una nicchia della navata laterale destra nella chiesa inferiore di San Clemente ${ }^{48}$, torna in Santa Maria Antiqua nella cappella di Teodoto (Fig. 6) realizzata durante il pontificato del greco Zaccaria (741-753) ${ }^{49}$ e nel pannello con l'iscrizione MARIA REGINA, già nell'atrio ora nella navata destra, voluto da papa Adriano I, di famiglia nobile romana (772-795). ${ }^{50}$ La ritroviamo ancora in S. Lorenzo fuori le mura, nella nicchia sud del nartece pelagiano. La decorazione conosciuta solo dalla docu-

46. ASTANT STYPENTES ANGELORUM PRINCIPES GESTARE NATUM... SD QUOD IPSE FACTUS EST, Bertelli 1964, 157.

47. Sui conflitti dogmatici ancora non risolti al tempo di Giovanni VII si veda Nordhagen 2002, 1757.

48. Per il dibattito critico e per la datazione alla metà del secolo si rinvia a Osborne 1981, 229310; Osborne 1984, 115-118; sulla interpreta- zione di questa nel quadro della "frammentazione liturgica”: Bauer 1999, 415.

49. Per un quadro critico e bibliografico complessivo si rinvia ad Andaloro 1987, 267-270. L'incidenza della devozione privata nelle immagini della cappella è stata approfondita da Belting 1987 e da Teteriatnikov 1993. Si veda anche con ulteriore bibliografia Bauer 1999, 408-410.

50. Osborne 1987, 195-197; Andaloro 1987, 274. 
mentazione ottocentesca (Figg. 7, 8) ${ }^{51}$ è stata recentemente riscoperta e studiata da Acconci. ${ }^{52}$

L'affresco frammentato (Figg. 9, 10) rinvenuto nel 1991 in un sarcofago in S. Susanna riproponeva il modello aulico della Madonna della Clemenza per una cappella privata. Insieme ad esso, oggi ricomposto, sono stati trovati nel sarcofago altri frammenti di intonaco dipinto appartenenti al medesimo oratorio: l'Agnello mistico tra S. Giovanni Battista e S. Giovanni Evangelista ed alcune teste di santi. Andaloro, con analisi stilistiche, riconduce questi pezzi all'VIII secolo avanzato e li attribuisce all'intervento di Adriano I nella chiesa. Lo stacco può essere ricondotto in base a considerazioni archeologiche intorno all'800. I frammenti sono di grande interesse sia per la qualità intrinseca dell'opera sia per il loro significato nella storia della religiosità: sono stati disposti nel sarcofago, la cui inumazione risale ad un'epoca precedente, in modo tale che se ne potessero percepire i nessi figurativi ${ }^{53}$.

Torniamo al nostro soggetto. Il dibattito storiografico ha lungamente discusso sulle origini della iconografia che trasferisce le insegne del potere terreno, che sono principalmente quelle degli imperatori bizantini, alla trascendenza divina ${ }^{54}$. Certo è che la maggior concentrazione di tali immagini si trova a Roma e soprattutto nell'VIII secolo. A Costantinopoli non ne abbiamo tracce e nell'orbita bizantina rimane solo il mosaico di VI secolo ritrovato nel 1967 a Durazzo..$^{55} \mathrm{Gli}$ studi sulla letteratura teologico-dottrinale e sulla liturgia asseriscono che il con-

51. Limmagine n. 8 è tratta dal manoscritto preparatorio della Histoire de l'Art di Séroux d'Agincourt: copia personale di lavoro dell'A. contiene incisioni, disegni autografi ed appunti.

52. Acconci 2002. Nelle due nicchie del nartece si estendeva una decorazione dedicata alla Vergine oggi in parte perduta. La nicchia meridionale, di cui oggi la Collezione Lanciani conserva l'acquarello eseguito da Virginio Vespignani durante i lavori della cripta di Pio IX (1862-1865), prevedeva nella lunetta di fondo Maria Regina tra S. Agata e S. Lorenzo, nella volta a botte e nelle pareti laterali la croce gemmata in un cielo stellato e due teorie di Santi. La nicchia settentrionale conserva nella lunetta di fondo Maria orante tra S. Ciriaca e S. Caterina, nella volta a botte e nelle pareti laterali la stessa decorazione della nicchia gemella. La studiosa ha ritrovato i consunti lacerti in situ e la documentazione fotografica conservata presso la Pontificia Commissione di Archeologia Sacra risalente al 1911: ritiene possibile posticipare al pontificato di Pasquale I (817-824) le pitture datate in precedenza su basi iconografiche alla metà dell'VIII.

53. Sugli scavi condotti dalla Soprintendenza Archeo- logica di Roma e sul ritrovamento degli affreschi si veda: Cecchelli 1999, in particolare 239-240; sulla fase altomedioevale e in particolare carolingia dell'edificio si rimanda anche per la bibliografia a Bonanni 2003; soprattutto 370; sugli affreschi si rinvia ad Andaloro 2003 (relazione al convegno del 1993); Andaloro 2001; 643-645; Andaloro 2002, 725. Per l'affresco dell'Agnus Dei si veda Lindsay Opie 2002, in particolare sull'uso iconodulo dei frammenti come reliquie, 18171818. Sull'atteggiamento "permissivo" di Adriano in relazione alle obiezioni franche sull'equivalenza affermata a Nicea II nel 787 tra icona e reliquia si veda Sansterre 1997, 118.

54. Sul problema si vedano: Bertelli 1964, 206-212; Andaloro 1972-1973; Nilgen 1981, in particolare 4-11; Bertelli 1983, 30; Andaloro 1983; Thérel 1984, anche per il precedente di S. Maria Maggiore, 225236; Osborne 1984, 119-125; Bertelli 1994, 210, 238 n. 36; Cutler 1994, 339; Andaloro 2000, 662-663.

55. Andaloro 1983 con bibliografia precedente. Per le obiezioni alla cronologia del mosaico si veda Bertelli 1994, 238 n. 36 con bibliografia; per la contrastante identificazione dell'immagine regale in Cristo basileus si veda Thérel 1984, 231. 


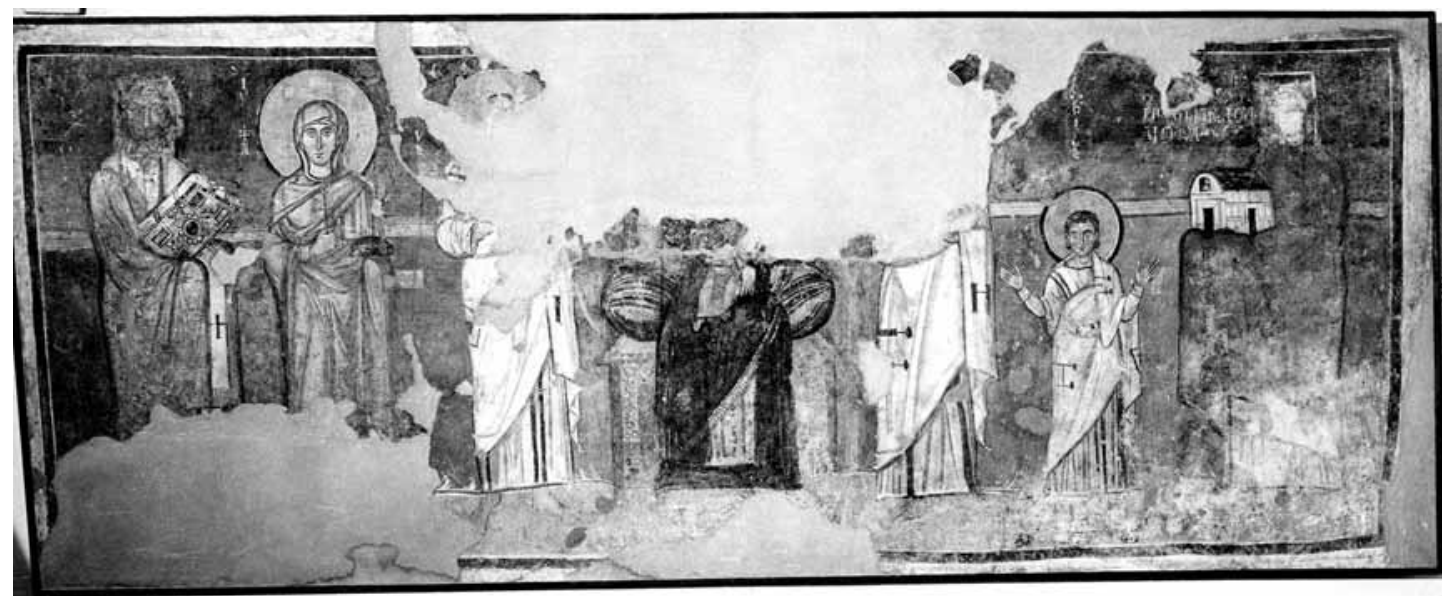

FIg. 6. Maria Regina tra i Santi Pietro, Paolo, Quirico e Giulitta, Teodoro primicerio e Zaccaria papa, Soprintendenza Archeologica di Roma (Foto Soprintendenza Speciale per il Polo Museale, neg. 197551)

cetto della regalità di Maria era già affermato nel VI secolo, sia nell'occidente latino che nell'oriente bizantino. ${ }^{56} \mathrm{Il}$ suo essere regina esprime la sua natura di Madre di Dio ed il suo ruolo eccezionale di regina dei cieli come mediatrice tra la divinità e l'umanità.

A Bisanzio il culto di Maria fu osteggiato dalla iconoclastia che dava vita in forma nuova alle eresie cristologiche: l'opposizione alla devozione mariana raggiunse il suo acme nel settimo decennio dell'VIII secolo. ${ }^{57}$

A Roma invece il culto di Maria si intensificò in parallelo agli sviluppi ufficiali della mariologia; la devozione crescente alla Vergine si accompagnava ad un moto antibizantino che non era più solo religioso ma diveniva in forma nuova politico -si affermava per la prima volta la distinzione tra sacerdotium ed imperium- ed economico. Bisanzio progressivamente usciva dall'orbita italiana sopraffatta dalle pressioni longobarde e dalla alleanza tra Roma e la nuova dinastia franca. ${ }^{58} \mathrm{Lim}$ magine di Maria Regina nell'VIII secolo è legata agli sviluppi della dottrina e della devozione inevitabilmente connessi alle dinamiche del conflitto iconoclasta e ed alle tensioni del nascente Stato della Chiesa. ${ }^{59}$ Lalba dell'età carolingia esalta sul

56. Si vedano essenzialmente: Andaloro 1983, 109111; Osborne 1984, 125, 140 n. 82.

57. Ostrogorsky 1968, 139-197. Sulla fase preparatoria alla restaurazione del culto delle icone, sulla cessazione degli attacchi contro il culto di Maria tra il 775 ed il 780 si veda $160-162$.

58. Bertolini 1941, 451-691; sul conflitto con Bisanzio che per la prima volta diventa anche economico: 431; sulla rottura politica tra Roma e Bisanzio: Ostrogorsky 1968, 150. Sulle "diverses étapes de la sécession romaine" Sansterre 1983 I, 127; II, 94 nota 302; 162 nota 137; sull'individuazione dell'anno 728 come "atto di morte del controllo bizantino su Roma” si veda Augenti 1996, 60 n. 66 con bibliografia.

59. Sulla acuta analisi della nuova immagine politica di Maria Regina quale espressione della Chiesa di Roma: Nilgen 1981; sulle immagini votive di Maria Regina: Thérel 1984, 233. 
piano figurativo la corrispondenza simbolica tra Maria e la Chiesa ${ }^{60}$ che la riflessione mariologica dei Padri aveva valorizzato soprattutto con Agostino. ${ }^{61}$

Le Marie Regine romane segnano un nodo in cui confluiscono l'insegnamento teologico, le tensioni spontanee del culto, la nuova ideologia romana del potere imperiale ${ }^{62}$ Esse anche nella loro formulazione stilistica costituiscono un progressivo allontanamento da Bisanzio. La Vergine con il Bambino del pilastro nord-ovest in Santa Maria Antiqua è ancora profondamente debitrice all'organicità antica; la prothesis di papa Zaccaria, malgrado le connessioni con il cantiere di Giovanni VII (Fig. 11), segna una tappa decisiva nel progresso della ricerca dell'astrazione. I bei volti femminili frammentati riemersi dopo dodici secoli dal sarcofago di S. Susanna siglano nella decisa riduzione alla bidimensionalità l'appartenenza irreversibile ad un nuovo orizzonte artistico e mentale.

\section{Episodi mariani nella scuola romana del mosaico carolingio}

L'oratorio vaticano dedicato a Maria voluto da Giovanni VII è l'ultimo esempio che possediamo di mosaico a Roma dell'VIII secolo. Sono perdute le opere di Zaccaria, Paolo I e Adriano I menzionate dalle fonti. ${ }^{63}$ Per nuove opere musive dobbiamo attendere il pontificato di Leone III (795-816): anche in questo caso comunque, al di là delle testimonianze documentali, di esistente rimane poco. ${ }^{64}$

Maria compariva nel mosaico absidale della chiesa di S. Susanna perduto con le trasformazioni di fine '500. La Vergine prendeva posto nel consueto schema teofanico di tradizione romana alla destra di Cristo seguita da Pietro, la martire titolare ed il papa committente, alla sinistra del Redentore si schieravano Paolo, i Santi Gaio e Gabico e la figura di Carlo Magno. Linserimento di Maria non costituiva una novità in assoluto: ricordiamo che era comparsa nel registro inferiore al centro del catino dell'oratorio lateranense e che con probabilità, su questo torneremo poco più avanti, campeggiava almeno su un'abside romana; nuova e tutta dettata dal momento storico era l'insistenza sui legami tra il pontefice ed il re dei franchi, sviluppata successivamente come è noto nei perduti mosaici lateranensi. ${ }^{65}$

60. Si veda soprattutto il Sermo in purificatione Sanctae Mariae di Ambrogio Autperto abate di San Vincenzo al Volturno (777-778) ed in particolare i paragrafi 2-4: P.L. 89, 1293-1295; sulle riflessioni mariologiche dell'abate di S. Vincenzo si vedano D'Onofrio 2000, 59. Sui rapporti tra l'opera di Autperto e la Maria Regina della cripta dell'abate Epifanio (824-842) nel monastero di San Vincenzo si vedano Thérel 1984, con estesa bibliografia, in particolare 235; Valente 1996, 85-137. Per una quadro sintetico della interpretazione teologica di Maria come Chiesa in età carolingia si veda Nilgen 1981, 22-23.

61. "Ecclesia sicut Maria et mater et virgo est": Agostino, De sancta virginitate liber unus, P.L.
40, 397. Si veda: Cecchelli 1946, 304; Simonetti 2000, 52.

62. Burgarella 2002, 981-983 con bibliografia.

63. L.P. I, 432, XVIII, 218; 464, IV, 259; 502-503, LVI, 329; per la bibliografia si veda: Curzi 1993, 23.

64. Per un quadro complessivo critico si rimanda a Andaloro 1987, 275-278.

65. Per la descrizione ed i disegni che riguardano comunque solo le figure di Leone III e di Carlo citiamo solo: Ms. vat. lat. 5407, 92 (con disegno), 95v, 96 (descrizione e disegno); Ms. vat. lat. 1054, 234235 (disegno e descrizione). Per la pubblicazione completa della documentazione si veda Waetzold 1964, nr. 1057-1059, figg. 556-558. Per una analisi critica con bibliografia si veda Nilgen 1999. 


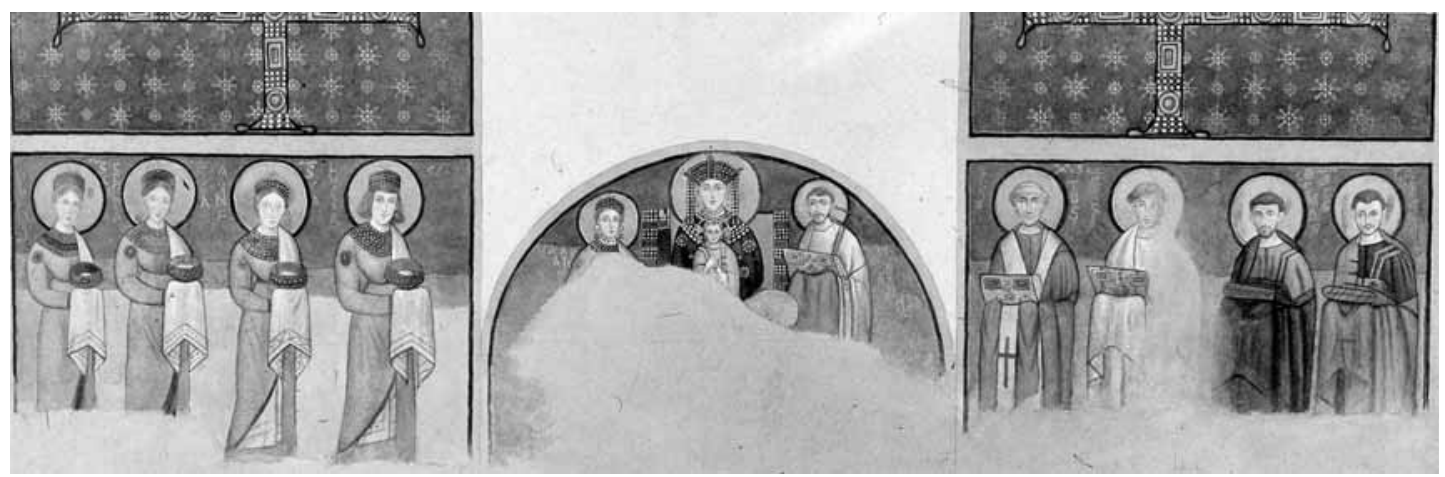

FIg. 7. Maria Regina con il Bambino tra Santi, disegno, Roma, Biblioteca di Archeologia e Storia dell'Arte, Collezione Lanciani, Roma XI, 45, 2, n. 31726 (Foto BIASA)

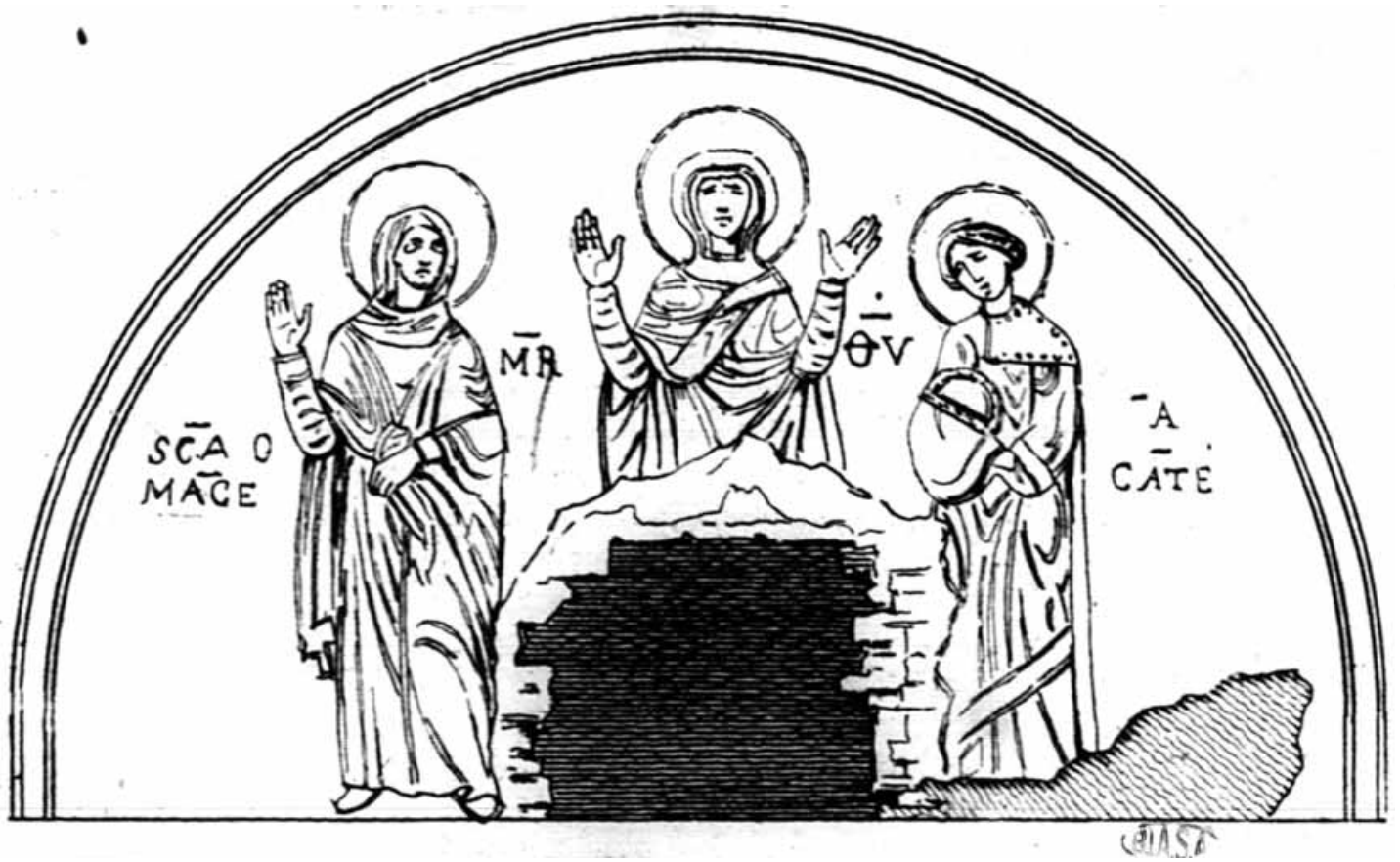

Fig. 8. Maria orante tra due Sante, Séroux d'Agincourt, Histoire de l'Art, Roma, Biblioteca di Archeologia e Storia dell'Arte, Collezione Lanciani, Ms. 103, tv. XI (Foto BIASA)

Maria ritorna nell'arco absidale della chiesa dei SS. Nereo e Achilleo: a sinistra nella scena dell'Annunciazione (Fig. 12), a destra nella figurazione di Maria con il Bambino ed un angelo (Fig. 13); nella parte centrale dell'arco prende posto la Trasfigurazione. Giunta ha identificato la chiave di lettura del programma iconografico in funzione anti-adozionista: per prima la studiosa ha svolto in modo completo la lettura iconografica ed iconologica delle immagini volte a "rappresentare il mistero della unione ipostatica dell'essenza divina e della natura 
umana nella Persona del Verbo Incarnato". ${ }^{66}$ L'eresia cristologica era nata in Spagna alla fine dell'VIII secolo: minava il dogma della Incarnazione e metteva in discussione la Divina Maternità di Maria; condannata ripetutamente, in particolare a Roma nel 798, si può considerare sconfitta, ma solo ufficialmente, nell'800. La trascrizione concettuale del mosaico appare limpida: le due raffigurazioni della Vergine esprimono l'idea della Incarnazione, la scena centrale con forza ribadisce la natura divina di Cristo. Anche il mosaico absidale, perduto alla fine del '500 con i restauri di Baronio, ma conosciuto nelle sue linee generali grazie ad un dipinto conservato nella Biblioteca Vaticana, si inserisce nell'unità complessiva: il tema aniconico della croce che campeggia su un drappo rappresenta la vittoria di Cristo. ${ }^{67}$

Il mosaico ci è giunto fortemente alterato dai restauri del 1832: solo quattro metri quadrati, secondo la ricostruzione di Matthiae, sono intatti. I due gruppi con Maria sono prevalentemente originali nelle parti superiori. ${ }^{68}$ Dal punto di vista dello stile assistiamo all'esordio di una nuova scuola ${ }^{69}$ : le tessere solo vitree, di formato piuttosto uniforme, si dispongono con una nuova irregolarità a costituire vivaci aree cromatiche guidate da una regia rigorosamente bidimensionale. Lo schema dell'impressionismo antico viene reinterpretato con una nuova sensibilità astratta: nella costruzione a macchie la dissociazione cromatica prevale sulla ricomposizione della forma.

Dedichiamoci a Pasquale I (817-824). Con questo pontefice come è noto abbiamo una organica produzione di opere musive. ${ }^{70}$ Osserviamole dal nostro punto di vista. Maria è protagonista nel catino di S. Maria in Domnica (figg. 14-15); compare quattro volte in Santa Prassede: nell'arco trionfale alla sinistra di Cristo nella città gemmata ${ }^{71}$ e tre volte nella preziosa cappella di San Zenone: nell'arco di ingresso con il Bambino; nella lunetta di fondo in figura di Deesis e nella lunetta di sinistra tra le Sante Prassede, Pudenziana e Teodora Episcopa. ${ }^{72}$

66. Giunta 1976, 196-197.

67. Sulle persistenze dell'adozionismo in Occidente tra l'800 e l'840 si veda Schmitt 1987, 278. Sulle obiezioni mosse all'interpretazione in chiave adozionista: Curzi 1993, 31. Sulle connessioni tra l'antiadozionismo e la dottrina del filioque: Peri 2000, 437-438.

68. Matthiae 1967, I, 514-515; II, grafico senza numero. Le fotografie rivelano con facilità anche nelle parti superiori riprese nei nimbi e nel collo del Bambino. Sull'ultimo restauro e sulla bibliografia relativa si rinvia a Curzi 1993, 22.

69. Per l'articolato panorama critico volto all'esame degli aspetti tecnici e degli innesti stilistici provenienti dall'oriente bizantino rinviamo a Curzi 1993, in particolare 25-26.
70. Sul ruolo storico di Pasquale I nella città di Roma si veda Krautheimer 1981, 161-173; per una rivisitazione del concetto di rinascenza carolingia a Roma: Ballardini 1999, 65-67; Pace 2002.

71. Matthiae 1967, I, 238; II, fig. 186.

72. Per lo status quaestionis del complesso di S. Prassede si rinvia ad Andaloro 1987, 280. Per il sacello di S. Zenone ricorderemo i fondamentali studi di Brenk 1972-1974, e di Davis-Weyer 1976 volti ad indagare la componente bizantina dell'iconografia. Per il piccolo mosaico di XIII secolo raffigurante la Madonna in trono con il Bambino tra le Sante Prassede e Pudenziana nella nicchia sopra l'altare si veda: Matthiae 1967, I, 385; Brenk 1972-1974, 220; Pace 2000, 107-108; Acconci 2002, 1802 n. 26. 


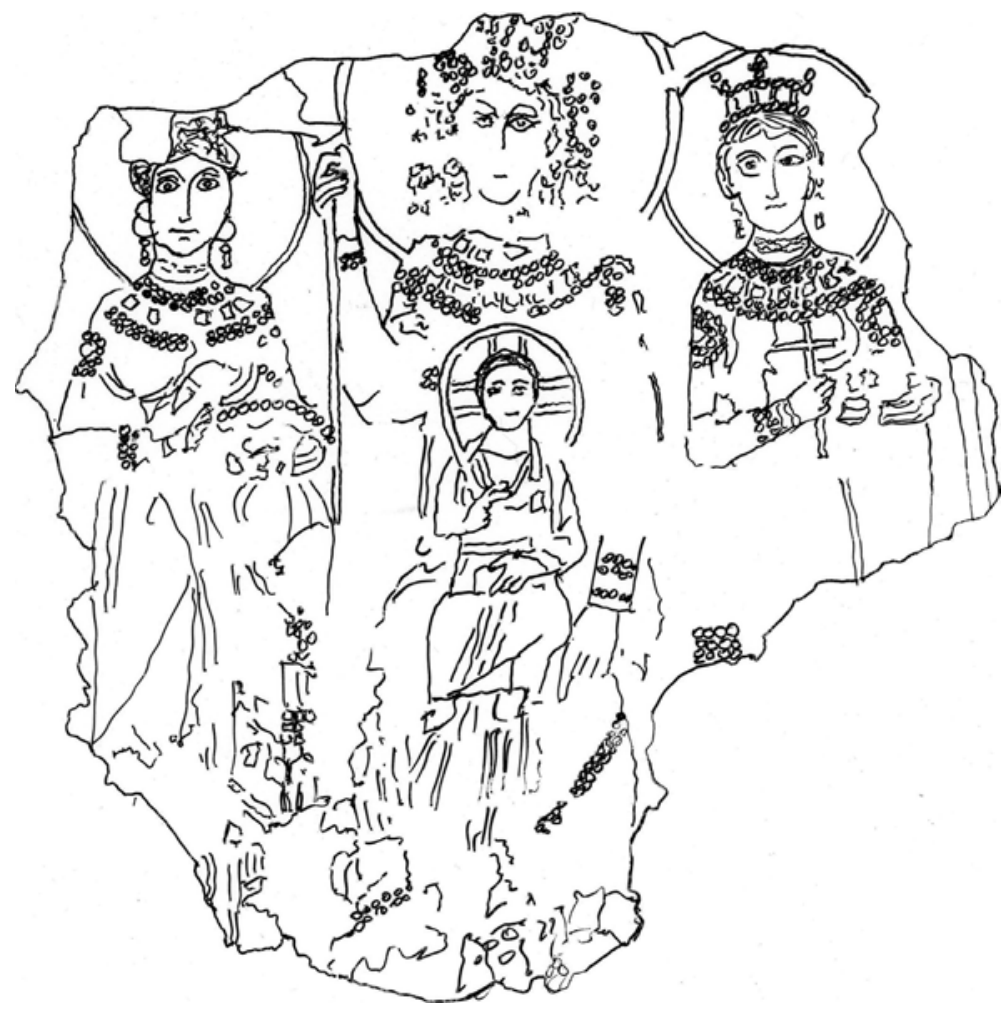

FIG. 9. Maria Regina con il Bambino fra Sante, Roma, Sacrestia di S. Susanna (disegno dell'a.)

FIG. 10. Maria (particolare), Roma, Sacrestia di S. Susanna (disegno dell'a.)
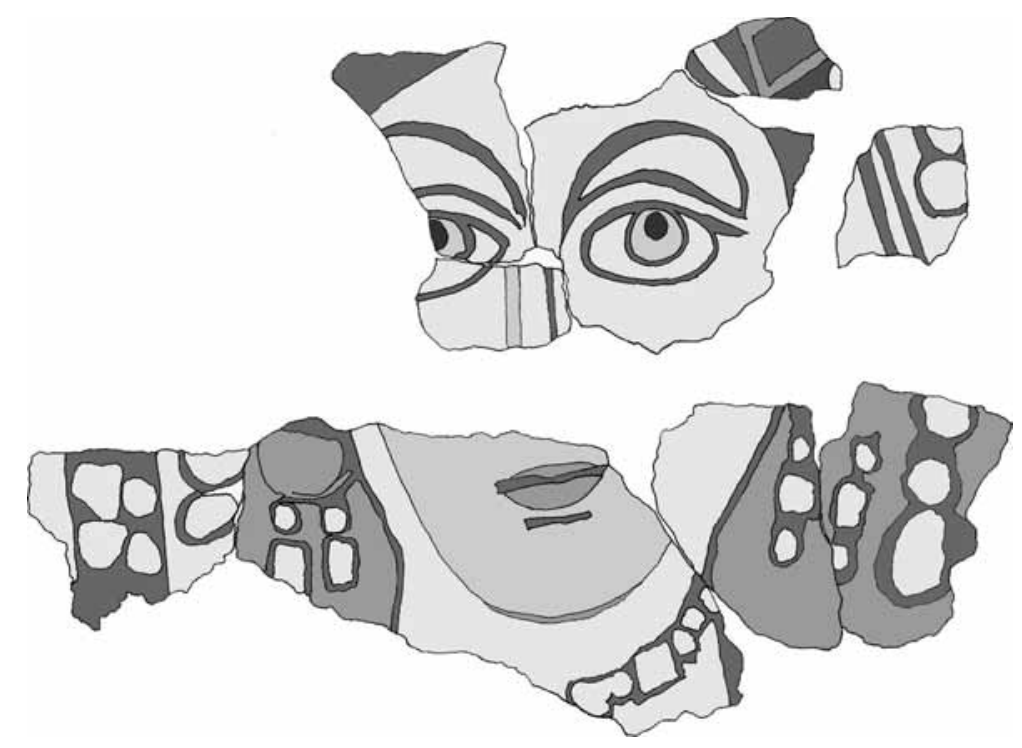


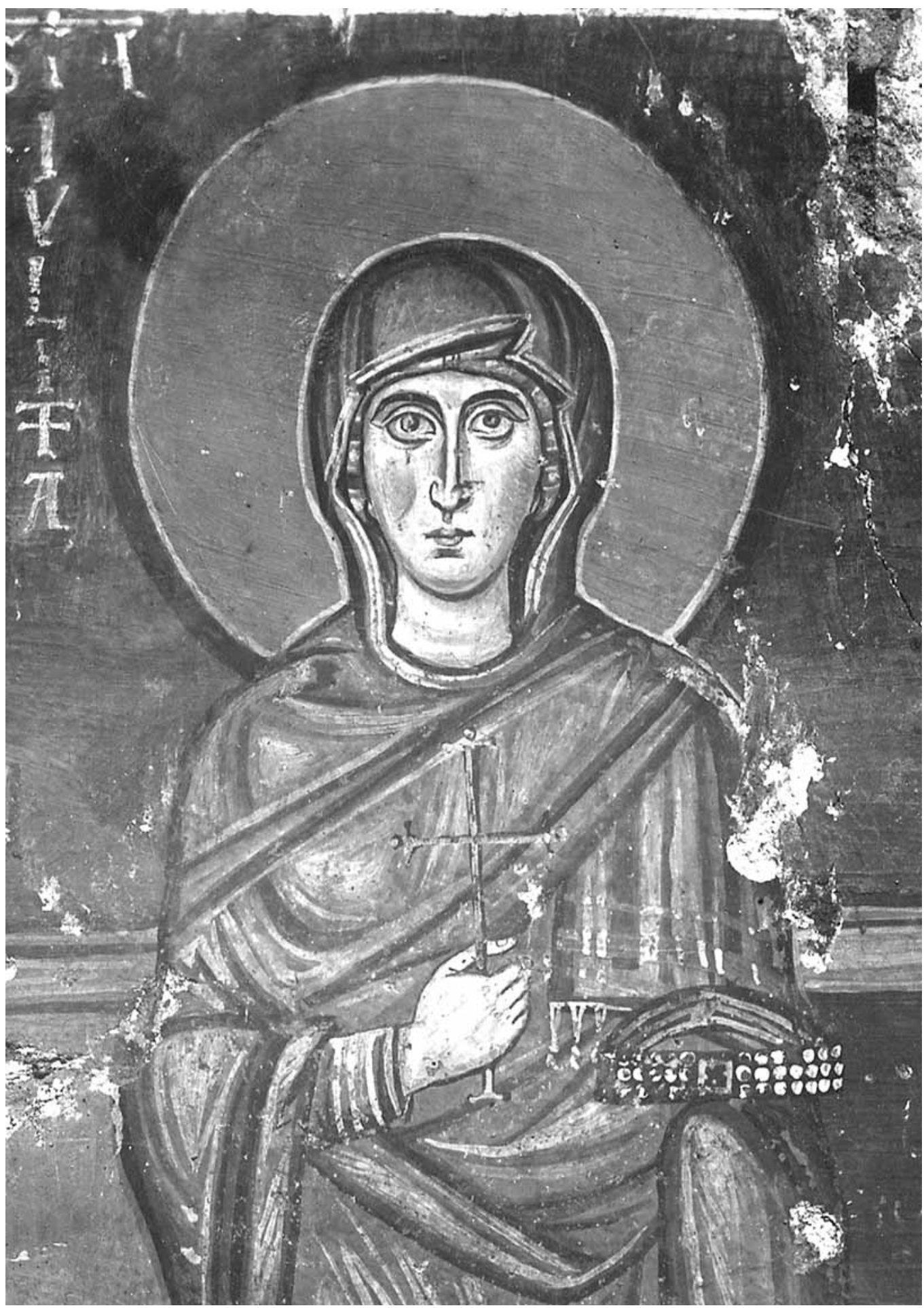

Fig. 11. S. Giulitta (particolare), Roma, Santa Maria Antiqua, cappella dei SS. Quirico e Giulitta, parete di fondo (Foto SAR, neg. 406) 
Nella chiesa di Santa Cecilia Maria Regina si presentava al colmo dell'arco trionfale (Fig. 16) perduto nei lavori settecenteschi del cardinale Acquaviva. ${ }^{73}$

Soffermiamoci sul catino di S. Maria in Domnica. La lettura stilistica proposta da Oakeshott lo indica come il primo della serie musiva del pontefice; l'analisi del Liber Pontificalis condotta da Ballardini colloca i lavori di ricostruzione a fundamentis della chiesa e della decorazione musiva tra l'818 e l'819, in parallelo alla analoga ristrutturazione in S. Cecilia ed alla fondazione in S. Prassede di un monastero maschile greco. ${ }^{74} \mathrm{Il}$ catino costituisce nella documentazione attualmente esistente una novità a Roma. Per la prima volta abbiamo a Roma Maria protagonista in un'abside: la Vergine ha gli attributi regali del trono gemmato, del cuscino purpureo e delle pantofole rosse ma la sua testa è coperta dal semplice maphorion. Tiene sulle sue ginocchia il Bambino, è circondata da due schiere compatte di angeli ed ai suoi piedi, anche questa è una novità per una decorazione absidale, Pasquale si inginocchia in gesto di proskunesis tra $\mathrm{i}$ papaveri, simbolo antico del Paradiso.

Come è noto non abbiamo una testimonianza diretta che attesti l'esistenza di absidi romane dedicate alla Vergine anteriori a S. Maria in Domnica. Beda il Venerabile ricorda che il suo maestro Benedetto Biscop nei primi decenni del VII secolo riportò dai suoi viaggi a Roma "picturas imaginum sanctarum" quali modelli per la decorazione della chiesa di S. Pietro nel monastero di Yarrow: tra gli schizzi figurava una immagine della Vergine usata per la conca absidale. ${ }^{75}$ Non sappiamo da dove provenisse il modello romano esportato nel VII secolo in Northumbria. La testimonianza settecentesca dell'erudito Mazzocchi ricorda per il V secolo, ma in modo non inequivocabile, la Vergine in trono nell'abside di S. Maria Maggiore. ${ }^{76}$ Per l'abside di S. Maria Antiqua come abbiamo visto esistono solo delle supposizioni. ${ }^{77}$

È nelle chiese greche invece, per quanto mutilate dalle distruzioni iconoclaste, che la Vergine occupa costantemente lo spazio absidale essendo riservato al Salvatore lo spazio privilegiato della cupola. ${ }^{78}$ La cellula compositiva utilizzata in S. Maria in Domnica viene dall'oriente bizantino dell'età preiconoclasta: citiamo l'abside di Parenzo (VI secolo) e quelle cipriote della Panagia Kanakaria a Lytrankomi (VI secolo) e della Panagia Angeloktistos (VII secolo?). ${ }^{79}$ Della fase

73. Sulla descrizione dell'arco si veda Ciampini 1699, 156-157. Per una ulteriore documentazione grafica si rinvia a Waetzold 1964, nr. 64, fig. 35. Per i frammenti esigui ritrovati nel sottotetto si veda: Matthiae 1970, 93.

74. Oakeshott 1967, 166; si veda anche Andaloro et al. 2002, 83; Ballardini 1999, 22.

75. Beda, P.L. 94, 718 A-B; Meer 1938, 17, 24, 386387; Meyvaert 1977, 66, 72-74; Thérel 1984, 117, n. 236; Christe 1996, 119-120.

76. Mazzocchi 1755, 705-706. Per la ipotesi rico- struttiva dell'abside di $\mathrm{V}$ secolo in S. Maria Maggiore in rapporto alla Basilica Suricorum in S. Maria in Capua Vetere si veda Cecchelli 1946, 306; Ihm 1960, 55-56, fig. 10, 132-133; Thérel 1984, 40, 100, n. 137; Andaloro et al. 2002, 100, fig. 77.

77. Si veda in questo testo n. 29.

78. Meer 1938, 324.

79. Si rinvia a Lazarev 1967, 85, 86, figg. 63, 66. Sulla Panagia Angeloktistos, fig. 52, e sulla sua discussa cronologia: 98 n. 18. 


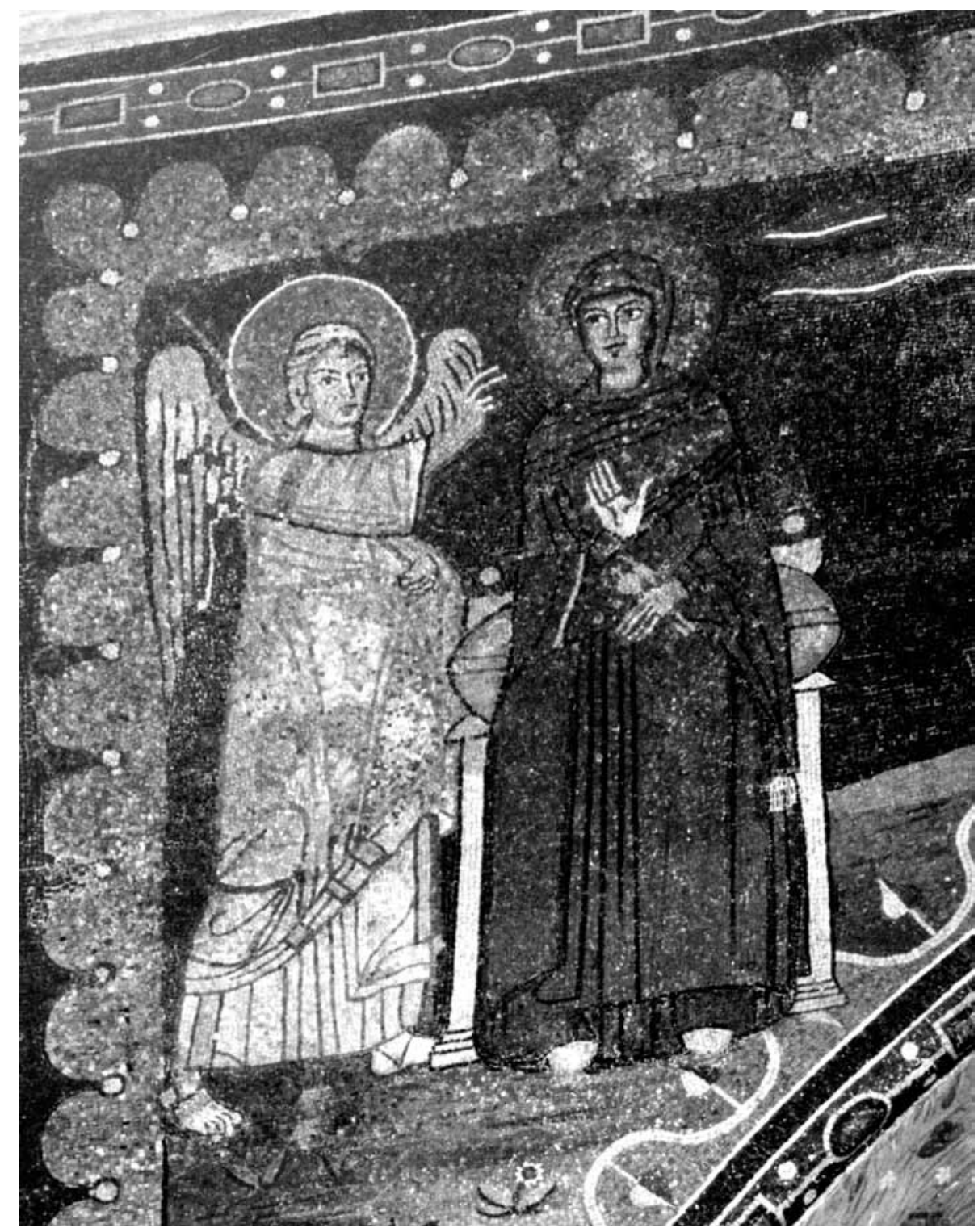

FIg. 12. Annunciazione, Roma, SS. Nereo e Achilleo, mosaico dell'arco absidale (Foto Soprintendenza Speciale per il Polo Museale, neg. 1777568)

post-iconoclasta ricordiamo solo che la perduta abside della Chiesa della Dormizione a Nicea presentava al centro Maria con il Bambino (787?, 843? ${ }^{80}$.

Notiamo che rispetto ai modelli bizantini l'abside di S. Maria in Domnica tende a riempire il catino con due folti gruppi di angeli disposti simmetrica-

80. Per l'analisi del complesso problema si rinvia anche per la bibliografia a Kitzinger 1992, 123-144. 
mente secondo la tradizione romana delle visioni apocalittiche. ${ }^{81}$ Inedita rispetto ai modelli orientali è anche la figura del pontefice inginocchiato che riprende nel gesto di venerazione l'immagine di Giovanni VII della icona di Santa Maria in Trastevere e quella di Teodoto del pannello dedicatorio nella cappella dei SS. Quirico e Giulitta in Santa Maria Antiqua (Fig. 17). ${ }^{82}$

Thérel e Wisskirchen hanno collegato la nuova immagine dell'abside celimontana con gli sviluppi della riflessione mariologica nella teologia dell'Assunzione. ${ }^{83}$ Andaloro e Thunø hanno individuato nella proskunesis che Pasquale I compie nel mosaico romano una chiave di lettura complementare: negli anni della restaurazione iconoclasta di Leone V l'Armeno (813-820) il pontefice si oppone rappresentando nel mosaico l'atto di devozione che si deve alle icone. Lo studioso danese suggerisce inoltre di identificare - sulla base della iscrizione absidale che paragona lo splendore del mosaico alla luce di Febo che vince la notte - la funzione originaria, neoplatonica, della monumentale "icona mariana": transito dalla forma all'archetipo. ${ }^{84}$

Aggiungiamo un passo della lettera che Pasquale I inviò a Leone V sulla questione delle immagini. La lettera, pervenutaci solo nel testo greco, pubblicata per la prima volta nel 1868 da Pitra da un manoscritto del Collegio Romano oggi perduto, riedita da Mercati nel 1905 dal codice ambrosiano H 257 inf., è rimasta poco conosciuta dalla storiografia specifica per la sua assenza dai repertori tradizionali delle fonti. Lo studio di Grumel del 1960 rimane fondamentale, dobbiamo invece ad Englen una recentissima trascrizione, che a giudizio della studiosa non si discosta comunque significativamente da quella di Mercati, e la prima traduzione completa in italiano. Grumel situa la cronologia della lettera tra l'817 e l'819 attraverso l'analisi di fonti parallele di provenienza bizantina ed individua il testo quale documento prezioso, l'unico rimasto nella letteratura occidentale, per comprendere le relazioni di quei due anni tra Roma e Bisanzio. La lettera inviata a Costantinopoli costituisce la ferma risposta del pontefice ai tentativi di compromesso di Leone $\mathrm{V}$ ed è a giudizio dello studioso forse il risultato di un sinodo romano di cui si sono perduti gli atti. ${ }^{85}$

81. Davis-Weyer 1966, 116. Il confronto con le schiere angeliche della Adorazione della Croce di Santa Maria Antiqua, è stato, a quanto mi risulta, proposto per la prima volta dalla studiosa, lo troviamo in seguito costantemente accolto. Sui due gruppi angelici delle visioni apocalittiche romane si veda anche Meer 1938, 324.

82. Andaloro et al. 2002, 83.

83. Thérel 1984, 234-235; Wisskirchen 1995-1997, in particolare 384-385, 388-389.

84. Andaloro et al. 2002, 83-84; Thunø 2002, 144145 con bibliografia precedente dell'A.; Thunø 2003, 94. I temi relativi alla questione delle immagini sono stati ripresi e approfonditi da
Svizzeretto 2003. Ballardini nel suo studio su Pasquale I , rimasto purtroppo privo della seconda parte, annunciava un "approccio semiotico” analogo a quello di Thunø: la studiosa prevedeva di "leggere le iconografie secondo la loro peculiarità stilistica di testi figurativi integrati al più complesso sistema testualizzato alto medioevale", Ballardini 1999, 6.

85. Pasquale I, ed Mercati 1905, con l'indicazione bibliografica di Pitra; Grumel 1960; Englen 2003. Si veda anche Thunø 2003, 135-137, 146147. Sul nuovo movimento iconoclasta, sul sinodo che sconfessò Nicea II si veda Ostrogorsky $1968,180-181$. 


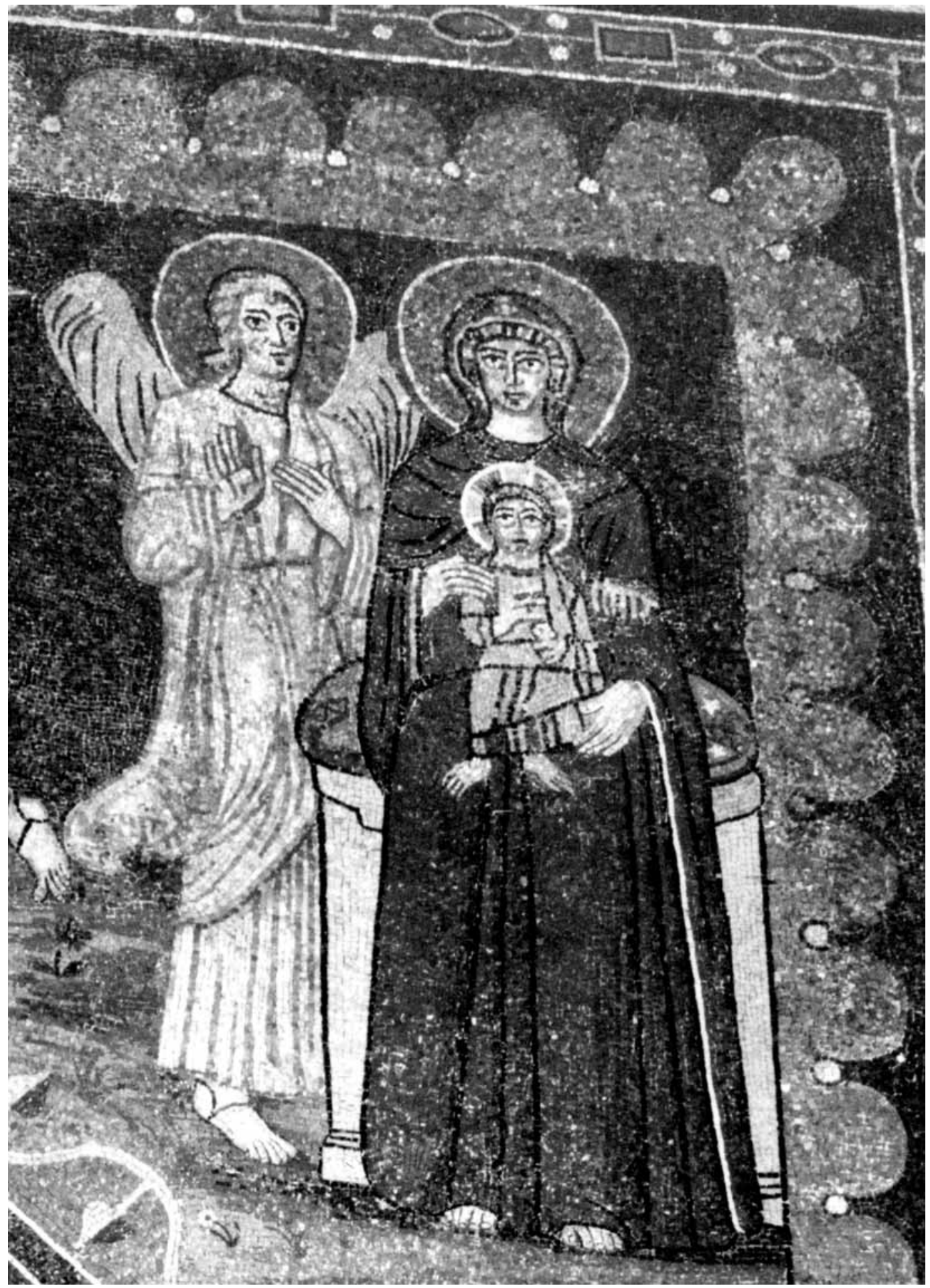

Fig. 13. Maria con il Bambino e un angelo, Roma, SS. Nereo e Achilleo, mosaico dell'arco absidale (Foto Soprintendenza Speciale per il Polo Museale, neg. 1777598) 


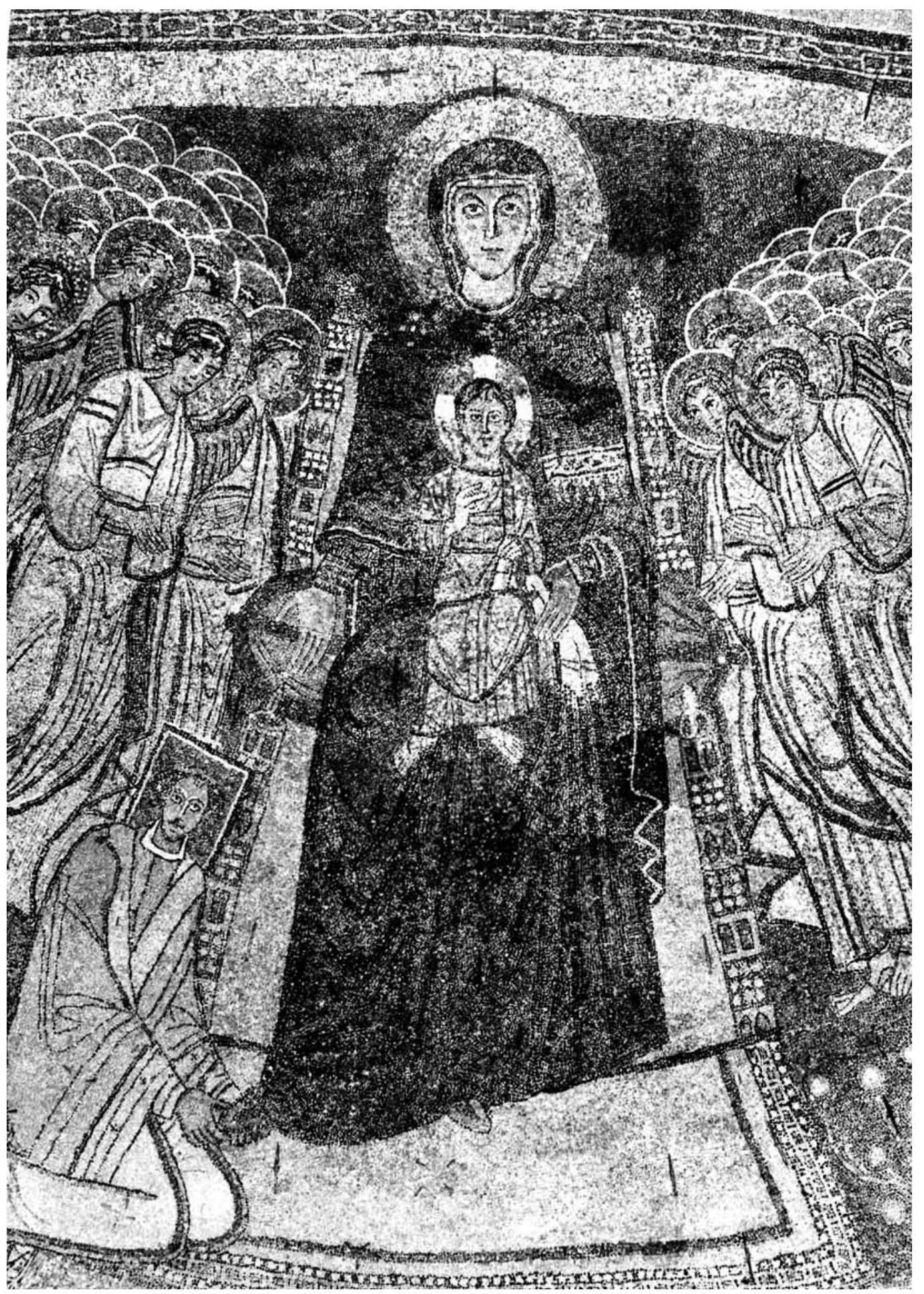

FIG, 14. Maria con il Bambino tra gli angeli e papa Pasquale I, Roma, S. Maria in Domnica, mosaico absidale (Foto Soprintendenza Speciale per il Polo Museale, neg. 138483) 


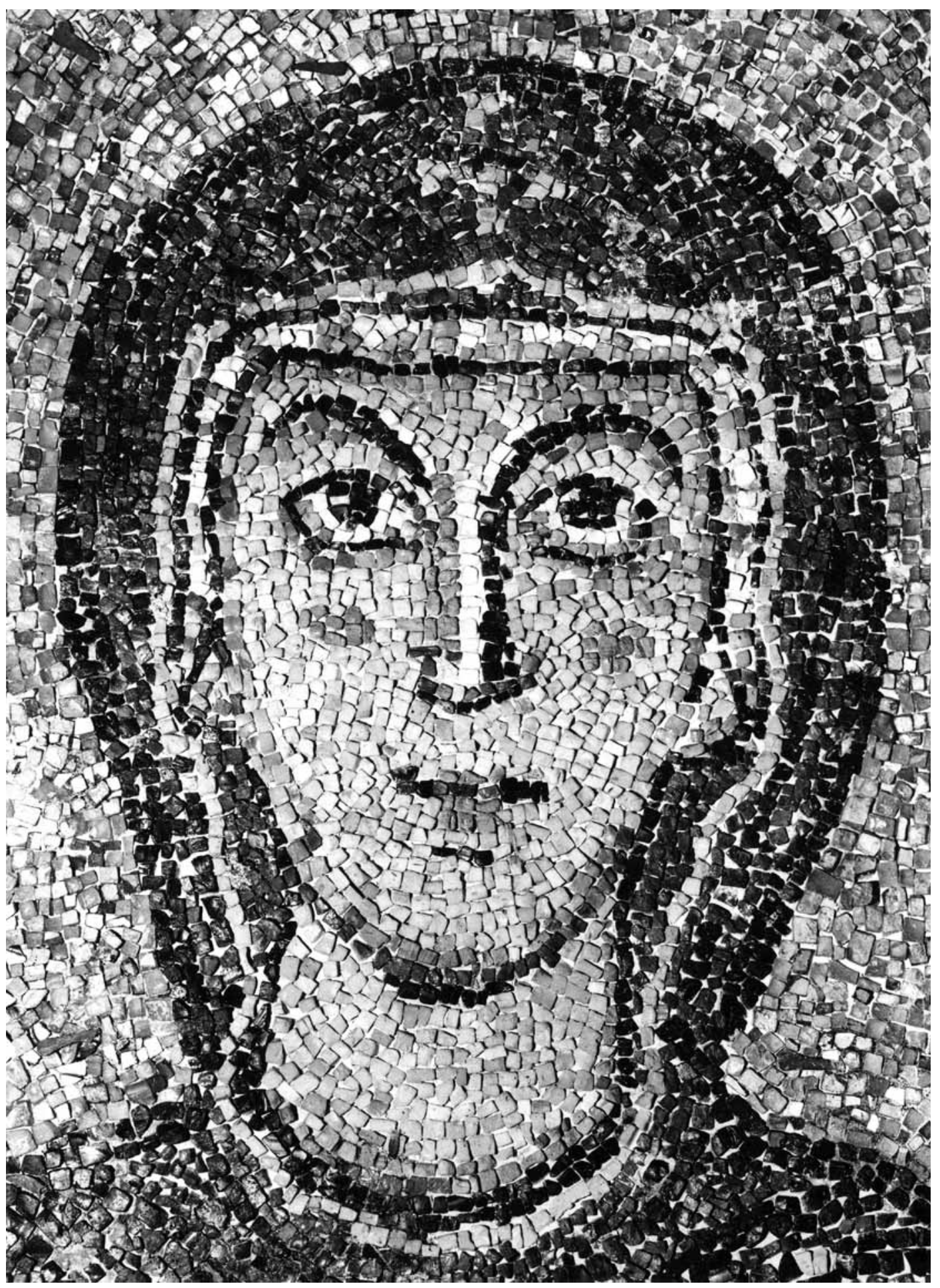

Fig. 15. Maria (particolare) Roma, S. Maria in Domnica, mosaico absidale (Foto Soprintendenza Speciale per il Polo Museale, neg. 138494) 


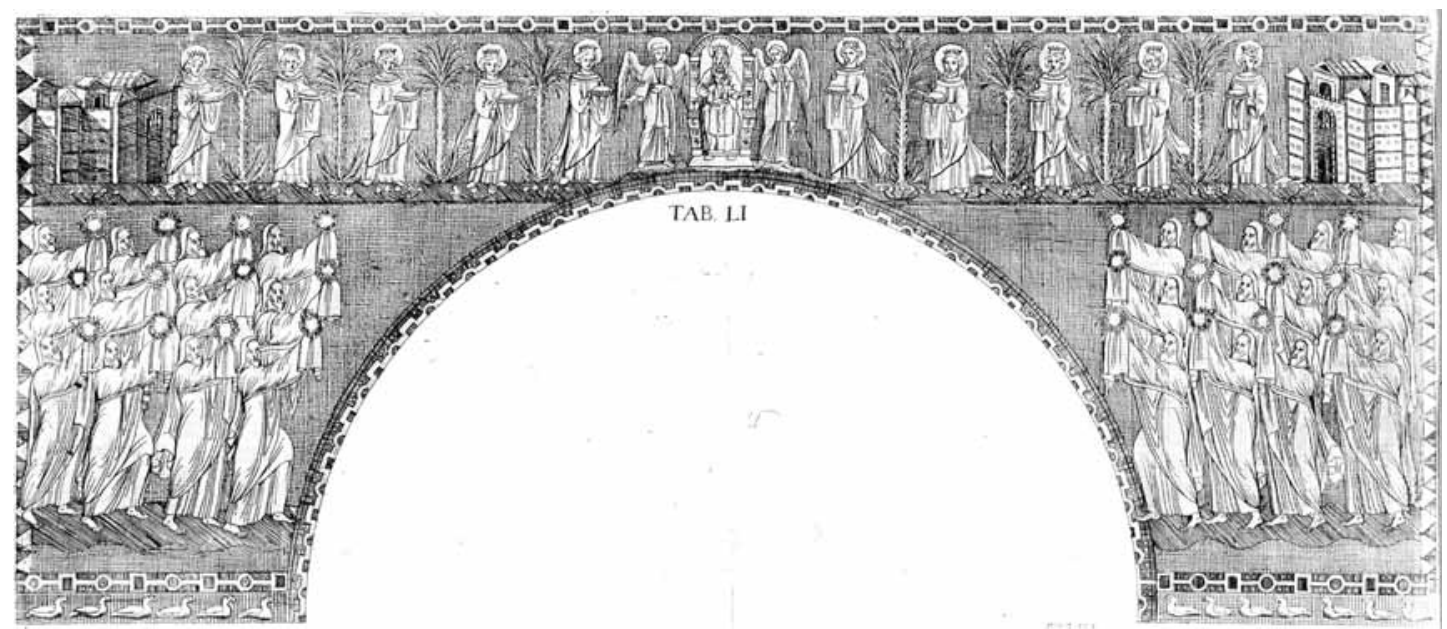

Fig. 16. Arco trionfale della chiesa di S. Cecilia, Ciampini, Vetera Monimenta, Roma 1699, II, tv. LI (Foto BIASA)

"3. Come intenderemo la preservazione della distinta e duplice essenza in Cristo se non lo vogliamo raffigurare secondo la natura umana nella quale egli ha sofferto? Noi infatti veneriamo la santa immagine di Cristo poiché il figlio essendo uno e indivisibile e avendo una sola sostanza, di cui si raffigura la carne, pur essendo evidentemente inseparabile dalla divinità indefinibile e indescrivibile può essere raffigurato e adorato". .86

Il dogma della Incarnazione è l'argomento che Pasquale I usa nella sua opposizione all'imperatore bizantino. Il mosaico romano raffigurando la Divina Maternità di Maria esprime il presupposto alla liceità del culto delle immagini.

Lo stile del mosaico, malgrado i prestiti bizantini di ordine compositivo ed iconografico, è romano. Il catino colmo di angeli non ha niente a che fare con lo spazio neutro della calotte bizantine. L'accentuato cromatismo a macchie, in tutto analogo in quello che abbiamo riscontrato nell'arco dei SS. Nereo e Achilleo, organizzato con piena scansione bidimensionale, rivela la propria estraneità alla cultura artistica bizantina e l'appartenenza all'orizzonte romano.

La cultura della città di Roma si allontana dal mondo greco, che pure in parte ancora ne segna la vita. La cancelleria papale si avvale di ellenofoni, prova ne è la lettera che abbiamo esaminato, il monastero fondato da Pasquale I in S. Prassede, forse la sua chiesa preferita dove fu sepolta la madre Teodora, risuona di notte e di giorno del canto delle lodi in lingua greca, pure la "tutela culturale" di quel mondo che ha toccato il suo culmine con il greco Zaccaria è nei primi decenni del IX secolo in declino ${ }^{87}$ : una nuova aristocrazia della terra, senza 


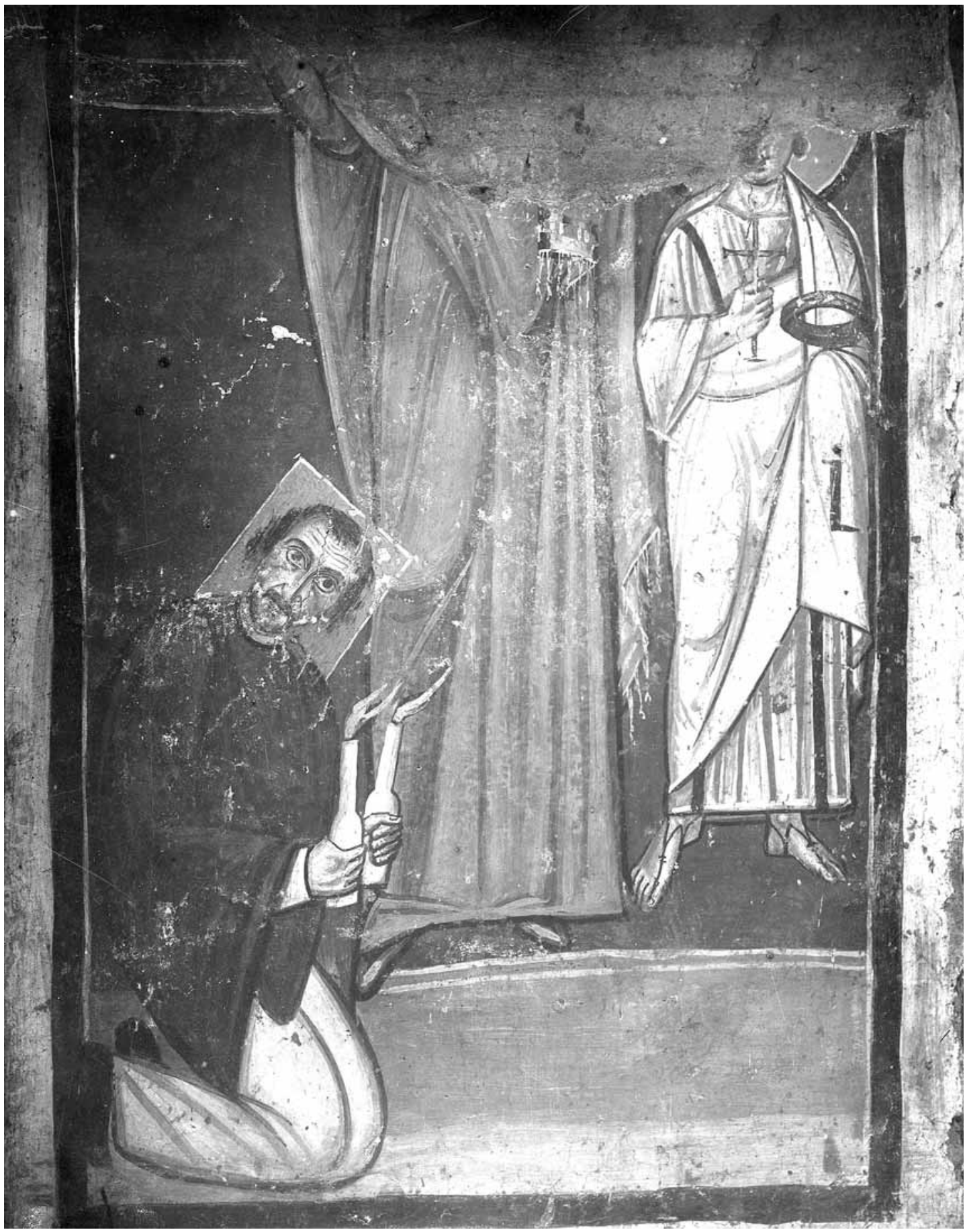

Fig. 17. Papa Zaccaria inginocchiato davanti ai Santi Quirico e Giulitta, Roma, Santa Maria Antiqua, Cappella dei SS. Quirico e Giulitta, parete a destra dell'ingresso (Foto SAR, neg. 632) 
legami con la amministrazione bizantina si faceva strada, il monotelismo e l'iconoclastia finirono per creare una attitudine negativa verso Bisanzio mentre la teologia latina costituiva la forza liberatrice della Roma papale. "Il greco, ma piuttosto la lingua che la cultura, fu abbandonato". .8

Se pensiamo al mosaico della Chiesa della Navicella troviamo qualcosa di analogo: è intimamente legato nella sua genesi al mondo bizantino eppure nuovo e scisso da legami con esso.

\section{Conclusioni}

I soggetti mariani esaminati rivestono un ruolo di "fossile guida": registrano con la loro presenza i conflitti legati prima alle eresie cristologiche poi alla crisi iconoclasta.

Dall'esame condotto notiamo come lo scontro con la politica religiosa di Bisanzio non costituisca a Roma nel VII secolo un elemento di separazione culturale dall'impero. A S. Venanzio assistiamo ad una compresenza di difficile decifrazione tra arte romana ed influssi dall'oriente bizantino; a S. Maria Antiqua ritroviamo un limpido capitolo "greco" anche nei momenti di maggiore opposizione all'impero.

È la metà dell'VIII secolo che dà l'avvio al processo di distacco da Costantinopoli ed all'inserimento di Roma con l'alleanza franca in un'orbita di gravitazione occidentale. Le Marie Regine non sono più solo una difesa dell'ortodossia, del credo duofisita e della legittimità delle immagini, ma anche una prima espressione politica della Chiesa di Roma: nelle soluzioni stilistiche troviamo un progressivo parallelo affrancamento dalla matrice bizantina.

Nel primi due decenni del IX secolo vediamo il processo giunto ad un punto di svolta: gli apporti dall'oriente bizantino sono ancora cospicui ma la sintassi formale autonoma è ormai costituita. L'opposizione di Pasquale I a Leone V nell'abside di Santa Maria in Domnica parla una lingua romana. La Vergine, gli angeli, il papa inginocchiato siglano nella inedita rielaborazione compositiva, nella disposizione delle tessere e del colore, nella preferenza tutta occidentale per la regia bidimensionale, una costruzione della forma che sarà destinata $\mathrm{a}$ segnare nella città di Roma un riferimento nell'età romanica.

Alessandra Themelly

Libera Università di SS. Maria Assunta

via Borgo S. Angelo 13

00193 Roma

88. Noble 1985, 62, per le altre osservazioni riportate: 61 . 


\section{BIBLIOGRAFIA}

Agostino, De sanctae virginitate liber unus, P.L. 40, 397610.

Ambrosii Autperti Opera. Sermo in purificatione Sanctae Mariae, P. L., 89, 1291-1304.

Beda Venerabilis, Vita sanctorum abbatum monasterii in Wiramutha et Girvum, P.L. 94, 713-730.

Ciampini G.. 1699: Vetera Monimenta, II, Roma.

Le Liber Pontificalis 1886: a cura di.L. Duchesne, I, Paris.

Mansi J.D. 1764: Sacrorum Conciliorum, X, Florentiae.

Mansi J-D. 1766: Sacrorum Conciliorum, XII, Florentiae.

Mazzocchi A.S.1755: Commentarii in marmoreum Neapolitanum Calendarium, III, Napoli .

Pasquale I ed. G. Mercati 1905: Lettera a Leone V l'Armeno, in Note di letteratura biblica e cristiana antica (Studi e testi, 5), 227-235.

Monumenta Spectantia 1894: Historiam slavorum meridionalium, XIV, Scriptores III, Zagreb.

Acconci A. 2002: "Note sulla decorazione pittorica altomedievale del nartece pelagiano di San Lorenzo fuori le mura", in Ecclesiae urbis, III, Roma, 1789-1812.

Andaloro M. 1970: "Note sui temi iconografici della Deesis e della Hagiosoritissa", RINASA, XVII, 85-153.

Andaloro M. 1972-1973: "La datazione della tavola di S. Maria in Trastevere", RINASA, XIX-XX, 139-215.

Andaloro M. 1983: "I mosaici parietali di Durazzo o dell'origine costantinopolitana della Maria Regina", in Studien zur Spätantiken und byzantinischen Kunst (Festschrift F.W. Deichmann) III, Mainz, 103-112.

Andaloro M. 1987: "Aggiornamento scientifico e bibliografia” in G. Matthiae, Pittura romana del Medioevo. Secoli IV-X., Roma, 213-330.

Andaloro M., 1989: "I mosaici dell'oratorio di Giovanni VII", in Fragmenta Picta (catalogo della mostra, Roma, Castel Sant'Angelo 1989-1990), 165-177.

Andaloro M. 1992: "Pittura romana e pittura a Roma da Leone Magno a Giovanni VII", in Committenti artistici e produzione libraria nell'alto medioevo occidentale (Atti della $39^{a}$ settimana di studio, Spoleto 1991) CISAM, II, 569-607.

Andaloro M. 2000, nn. 375-378, in Aurea Roma. Dalla città pagana alla città cristiana, (catalogo della mostra a cura di S. Ensoli ed E. La Rocca, Roma Palazzo delle Esposizioni), Roma, 660-663.

Andaloro M. 2001: "S. Susanna. Gli affreschi frammentati”, in Roma dall'antichità al medioevo. Archeologia e storia nel Museo Nazionale Romano Crypta Balbi, a cura di M.S. Arena, et al., Venezia, 643-645.

Andaloro M. 2002:"Le icone a Roma in età preiconoslasta”, in Roma fra Oriente e Occidente, (Atti della 49a settimana di studio, Spoleto 2001), CISAM, II, 719-753.
Andaloro M et al. 2002: Arte e iconografia a Roma. Dal tardo antico alla fine del Medioevo, Roma-Milano.

Andaloro M. 2003: "I dipinti murali depositati nel sarcofago nell'area di S. Susanna a Roma”, in 1983-1993. dieci anni di archeologia cristiana in Italia (Atti del VII Congresso Nazionale di Archeologia Cristiana, Cassino, 1993), a cura di E. Russo, Cassino, 377-383.

Armellini M. 1942: Le chiese di Roma dal XIV al XIX secolo. Nuova edizione con aggiunte inedite dell'autore, Illustrazioni a cura di C. Cecchelli, I-II, Roma.

Augenti A. 1996: Il Palatino nel Medioevo. Archeologia e topografia (sec. VI-XIII) (Bullettino della Commissione Archeologica Comunale di Roma- Supplementi 4)

Ballardini A. 1999: "Dai gesta di Pasquale I secondo il Liber Pontificalis ai monumenti iconografici delle basiliche romane di Santa Prassede, Santa Maria in Domnica e Santa Cecilia in Trastevere (I parte)", ASRSP, 122, 5-68.

Bauer F.A. 1999: "La frammentazione liturgica nella chiesa romana del primo Medioevo", RAC, II, 386-445.

Belting H.1987:, "Eine Privatkapelle im frühmittelalterlichen Rom", DOP, 41, 55-69.

Bertelli C.: 1964: "La Madonna di S. Maria in Trastevere”, BICR, 41-44.

Bertelli C.: 1983: "Traccia allo studio delle fondazioni medievali dell'arte italiana", in Storia dell'arte italiana, 5, Torino, 5-163.

Bertelli C.1994: "La pittura medievale a Roma e nel Lazio", in La Pittura in Italia. L'Altomedioevo, a cura di C. Bertelli, 206-242.

Bertolini O. 1941: Roma di fronte a Bisanzio e ai Longobardi (Storia di Roma IX), Roma.

Bertolini O. 1958: "Riflessi politici nelle controversie religiose con Bisanzio nelle vicende di VII secolo", in Caratteri del VII secolo in Occidente, (Atti della $5^{\text {a }} \mathrm{di}$ studio, Spoleto 1957) CISAM, II, 733-789.

Bisogni F. 2000: "L'iconografia di Maria”, in Maria Vergine, Madre, Regina, 161-169.

Bognetti G.P. 1955: "I rapporti etico politici fra Oriente ed Occidente dal V all'VIII secolo", in Storia del Medioevo (Atti del X Congresso Internazionale di Scienze Storiche), III, Firenze, 3-65.

Bonanni A. 2003: "Scavi e ricerche in S. Susanna a Roma. Le fasi paleocristiane, e altomedievali”, in 19831993: dieci anni di archeologia cristiana in Italia (Atti del VII Congresso Nazionale di Archeologia Cristiana, Cassino 1993, a cura di E. Russo), 359-375.

Bovini G. 1971: "I mosaici di San Venanzio a Roma", Corsi di cultura sull'arte ravennate, 18, 141-154.

Brenk B. 1972-1974: "Zum Bildprogramme der Zeno Kapelle in Rom" Archivo Español de Arqueologia, 45-47, 213-221. 
Brenk B. 2003: "Kultgeschichte versus stilgeschichte: von der <raison d'être > des bildes im 7. Jahrundert in Rom", in Uomo e spazio nell'alto medioevo (Atti della $50^{\text {a }}$ settimana di studio, Spoleto 2002), CISAM, II, 9711053.

Brenk B. 2004: "Papal Patronage in a Greek Church in Rome", in S. Maria Antiqua al Foro Romano, 67-81.

Brubaker L. 2004: "100 Years of Solitude: Santa Maria Antiqua and the History of Byzantine Art History", in S. Maria Antiqua al Foro Romano, 41-47.

Burgarella F. 2002: "Presenze greche a Roma: aspetti culturali e religiosi", in Roma fra oriente e occidente (Atti della $49^{\circ}$ settimana di studio, Spoleto 2001) CISAM, II, 941-988.

Caelius I. Santa Maria in Domnica, San Tommaso in Formis e il Clivus Scauri 2003: a cura di A. Englen (Collana Palinsesti Romani I), Roma.

Cecchelli C. 1946: Mater Christi, I, Roma.

Cecchelli M. 1999: "Dati da scavi recenti di monumenti cristiani”, MEFRM, 111, 227-251.

Christe Y. 1996: L'apocalypse de Jean. Sens et développements de ses visions synthétiques (Bibliothèque des Cahiers Archéologique XV), Paris.

Clédat M.-J. 1904: "Nouvelles recherches à Baouît", Comptes Rendus de l'Académie des Inscriptions et Belles-Lettres, 517-526.

Clédat M.-J. 1904-1906, "Le monastère et la nécropole de Baouît", Mémoires de l'Institut français d'Archéologie orientale du Caire, XII.

Conte P. 1971: Chiesa e primato nelle lettere dei papi nel VII secolo, Milano.

Curzi G. 1993: "I mosaici dell'arco absidale della chiesa dei SS. Nero e Achilleo", $A M, 2$, 21-45.

Curzi G. 1998: "I mosaici dell'oratorio di San Venanzio nel battistero lateranense: problemi storici e vicende conservative" (Atti del V Colloquio, AISCOM) Roma, 267-292.

Cutler A. 1994: "La <questione bizantina> nella pittura italiana: una visione alternativa alla <maniera greca >, in La Pittura in Italia. L'Altomedioevo, a cura di C. Bertelli, 335-368.

Davis-Weyer C 1966: "Die Mosaiken Leos III und die Anfänge der karolingischen Renaissance in Rom”, Zfkg, 29,1, 111-132.

Davis-Weyer C. 1976: “Die ältesten Darstellungen der Hadesfahrt Christi, das Evangelium Nikodemi und ein Mosaik der Zeno-Kapelle", in Roma e l'età carolingia (Atti delle giornate di studio, Istituto di Storia dell'arte della Università di Roma, 1976), Roma, 183-194.

De Rossi G.B. 1872: Musaici delle chiese di Roma anteriori al XV secolo, Roma.

D’Onofrio G. 2000: "Il mysterium Mariae nella teologia e nella pietà dell'alto medioevo latino", in Maria Vergine Madre Regina, Milano, 55-68.

Dyggve E., 1951: History of Salonitan Christianity, Oslo.

Ecclesiae urbis 2002: Atti del congresso internazionale di studi sulle chiese di Roma, a cura di F. Guidobaldi e A. Guiglia, I-III, Roma.
Englen A. 2003: "La difesa delle immagini intrapresa dalla chiesa di Roma nel IX secolo" in Caelius I, 257283.

Giunta D. 1976: "I mosaici della basilica dei SS. Nereo e Achilleo", in Roma e l'età carolingia (Atti delle giornate di studio, Istituto di Storia dell'arte della Università di Roma, 1976), Roma, 195-200.

Grabar A. 1946: Martyrium. Recherches sur le culte des reliques et l'art chrétien antique, II, Iconographie.

Grabar A. 1958: Les ampoules de Terre Sainte (MonzaBobbio), Paris.

Grabar A. 1968: Christian Iconography: A study on its Origins, Princeton.

Grumel V. 1928: "Recherche sur l'histoire du monothélisme", Echos d'Orient, XXVII, 257-277.

Grumel V. 1929: "Recherche sur l'histoire du monothélisme", Echos d'Orient, XXVIII, 272-282.

Grumel V. 1930: "Recherche sur l'histoire du monothélisme", Echos d'Orient, XXIX, 16-28.

Grumel V. 1960: "Les relations politico-religieuses entre Byzance et Rome sous le Règne de Léon V l'Arménien”, Revue des Etudes Byzantines, XVIII, 19-44.

de Grüneisen W. 1911: Sainte Marie Antique, Rome.

Iacobini A. 2000: Visioni dipinte. Immagini della contemplazione di Bawit, Roma.

Ihm Ch. 1960: Die Programme der christlichen Apsismalerei: vom 4 Jahundert bis zur Mitte des Jahrundert (Forschungen zur Kunstgeschichte und Christlichen, Archäologie, 4).

Kitzinger E. 1934: Römische Malerei vom Beginn des 7. bis zur Mitte des 8. Jahrunderts, Münich.

Kitzinger E. 1989: L'arte bizantina. Correnti stilistiche dell'arte mediterranea dal III al VII secolo, Milano.

Kitzinger E. 1992: Il culto delle immagini. L'arte bizantina dal cristianesimo delle origini alla iconoclastia, Firenze.

Krautheimer R. 1981: Roma. Profilo di una città. 3121308, Roma.

Lazarev V. 1967: Storia della pittura bizantina, Torino.

Maria Vergine Madre Regina: le miniature medievali e rinascimentali 2000: catalogo della mostra a cura di C. Leonardi e A. Degl'Innocenti (Roma Biblioteca Vallicelliana) Milano.

Lindsday Opie J. 2002: “Agnus Dei”, in Ecclesiae urbis, III, Roma, 1814-1862.

Matthiae G. 1967: Mosaici medioevali delle chiese di Roma, I-II, Roma.

Matthiae G.. 1970: S. Cecilia (Le chiese di Roma illustrate 113), Roma.

Meer van der F. 1938: Maiestas Domini. Théophanie de l'Apocalypse dans l'art chrétien (Studi di antichità cristiana. Pontificio Istituto di Archeologia Cristiana, XII), Rome-Paris.

Meyvaert P. 1979: "Beda und the Church Painting of Wearmouth-Yarrow”, Anglo-Saxon-England, 8, 63-77.

Nilgen U. 1981: "Maria Regina. Ein Politischer Kultbildtypus?”, RömJhkuGe 19, 3-33.

Nilgen U. 1999: "Die römischen Apsisprogramme der Karolingischen Epoche Päpstliche Repräsentation un 
Liturgie", Kunst und Kultur der Karolingerzeit, III, Paderborn, 542-549.

Noble Th.F.X. 1985: “The Declining Knowledge of Greek in Eight-and Ninth-Century Papal Rome”, Byzantinische Zeitschrift, LXXVIII, 56-62.

Nordhagen P.J. 1962a: "The earliest decorations in S. Maria Antiqua and their date", ActaAArtHist I, 53-72.

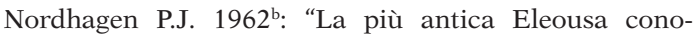
sciuta", Bollettino d'Arte, 47, 348-357.

Nordhagen P.J. 1963: "Icons designed for the disply of sumptuous votive gift", Bollettino d'Arte, 28, 351-353.

Nordhagen P.J. 1965: "The mosaics of John VII (705-707 A.D.) The mosaics fragments and their technique", ActaAArtHist, II

Nordhagen P.J. 1968: "The frescoes of Giovanni VII", ActaAArtHist, III.

Nordhagen P.J. 1978: "The frescoes of seventh century", ActaAArtHist, VIII, 89-142.

Nordhagen P.J. 1983: "S. Maria Antiqua Revisited", $A M$, I, 49-51.

Nordhagen P.J, 1997: "Mosaico", EAM, VIII, Roma, 563574.

Nordhagen P.J. 2000: "Constantinople on the Tiber: The Byzantines in Rome and the Iconography of their Images", in Early medieval Rome \& the Christian West. Essays in Honour of Donald A. Bullough, ed. by M.H. Smith, Leiden, 117-134.

Nordhagen P.J. 2002: "Early medieval church decoration in Rome and <the battle of images>, in Ecclesiae urbis, III, 1749-1762.

Oakeshott W. 1967: I mosaici di Roma, Milano.

Osborne J. 1981: "Early Mediaeval painting in San Clemente, Rome: The Madonna and Child in the niche", Gesta, 20, 229-310.

Osborne J. 1984: Early Mediaeval Wall-Paintings in the Lower Church of San Clemente, London.

Osborne J. 1987: "The atrium of S. Maria Antiqua, Rome: a history of art", PBSR, 55, 186-223.

Ostrogorsky G. 1968: Storia dell'impero bizantino, Torino.

Pace V. 2000: "Cristo-Luce a Santa Prassede", in Arte a Roma nel Medioevo. Committenza, ideologia e cultura figurativa in monumenti e libri, Napoli, 105-123.

Pace V. 2002: "La <Felix culpa> di Richard Krautheimer: Roma Santa Prassede e la <rinascenza carolingia>", in Ecclesiae urbis, I, 65-72.

Pelliccioni G. 1973: "Le nuove scoperte sulle origini del battistero lateranense", Memorie della Pontificia Accademia Romana di Archeologia, XII.

Peri V. 2000: “Il filioque divergenza dogmatica?", in Tempus implendi promissa, a cura di E. Reinhardt, Pamplona, 435-464.

Rushfort G. 1902, "The church of S. Maria Antiqua", PBSR, 1, 1-123.

Sansterre J.-M. 1983: Les moines grecs et orientaux à Rome aux époques byzantines et carolingienne (milieu $d u V I^{e}$ s.-fin $d u I X^{e} s$.), I, II, Bruxelles.
Sansterre J.-M. 1997: “Entre koinè méditerranéenne influences byzantines et particularités locales: le culte des images et ses limites à Rome", in Europa medievale e mondo bizantino a cura di G. Arnaldi e G. Cavallo (Nuovi studi storici 40), Roma, 109-124.

Santa Maria Antiqua al Foro Romano cento anni dopo 2004: Atti del colloquio internazionale (Roma 2000), a cura di J. Osborne, J. Brandt, G. Morganti, Roma.

Schmitt J.-Cl 1987: "L'occident, Nicée II et les images du VIII" au XIII' siècles", in Nicée II 787-1987. Douze siècles d'images religieuses (Actes du colloque. Paris 1986), Paris, 270-301.

von Schönborn Ch. 1975: "La primauté romaine vue d'Orient pendent la querelle du monoénergisme et du monothélisme", Istina, 20, 476-490.

Sensi M. 2000: "I santuari mariani", in Maria Vergine Madre Regina, 95-101.

Simonetti M. 2000: "Maria nei Padri greci e latini”, in Maria Vergine Madre Regina, 43-53.

Svizzeretto F. 2003: "Il mosaico absidale manifesto iconodulo: proposta di interpretazione", in Caelius I, 241256.

Tea E. 1937: La basilica di Santa Maria Antiqua, Milano. Teteriatnikov N. 1993: "For Whom is Theodotus Praying? An interpretation of the Program of the Private Chapel in S. Maria Antiqua”, CahArch, 41, 37-46.

Themelly A. 1999: "Il mosaico di San Venanzio in Laterano (640-649). Arte romana e influssi dall'oriente bizantino negli anni della crisi monotelita", Romanobarbarica 16, 317-345.

Thérel M.L. 1984: Le triomphe de la Vierge Église, Paris.

Thunø E. 2002: Image and Relic. Mediating the Sacred in Early Medieval Rome, Rome.

Thunø E. 2003: "The Cult of Virgin, Icons and Relics in Early Medieval Rome. A Semiotic Approach", ActaAArtHist, XVII, 79-98.

Valente F. 1996: S. Vincenzo al Volturno. Architettura ed arte (Miscellanea Vulturnense 1), Abbazia di Montecassino.

Velmans T et al. 1999: Rayonnement de Byzance, Paris.

Waetzold S. 1964, Die Kopien des 17. Jahrunderts nach Mosaiken und Wandemalereien in Rom, (Römische Forschungen der Bibliotheca Hertziana 18), WienMünchen

Wisskirchen R. 1990: Das mosaikprogamm von S. Prassede in Rom. Ikonographie und ikonologie (Jahrbuch für antike und Christentum, 17) Münster.

Wisskirchen R. 1995-1997: "Santa Maria in Domnica. Überlegungen zur frühesten apsidalen Darstellung der thronenden Maria in Rom", Aachener Kunstblätter 61, 381-393.

Zocca E. 1992: "Onorio e Marino due papi di fronte al monotelismo", in Martino I (649-653) e il suo tempo (Atti del XXVIII Convegno storico internazionale Todi 1991) Spoleto, 103-147. 
ActaAArtHist, Acta ad Archaeologiam et Artium Historiam Pertinentia

AM, Arte Medievale

AISCOM, Associazione Italiana per lo studio e la conservazione de mosaico

ASRSP, Archivio della Società romana di storia patria

BICR, Bollettino dell'Istituto Centrale del Restauro

CahArch. Cahiers Archéologiques

CISAM, Centro italiano di studi sull'alto medioevo, Spoleto
DOP, Dumbarton Oaks Papers

EAM, Enciclopedia dell'Arte Medievale

MEFRM, Mélanges de l'Ecole Française de

Rome- Moyen-Age

PBSR, Papers of the British School of Rome

RAC, Rivista di Archeologia Cristiana

RINASA, Rivista dell'Istituto Nazionale di Archeologia e Storia dell'Arte

RömJhkuGe, Römisches Jahrbuch für Kunstgeschichte

Zfkg, Zeitschrift für Kunstgeschichte 